Short title: Negative regulatory mechanism of $T a B \ln 1$

\title{
TaBln1 negatively regulates wheat resistance to stripe rust by reducing $\mathrm{Ca}^{2+}$
} influx

Shuangyuan Guo, ${ }^{2,3}$ Yanqin Zhang, ${ }^{1,3}$ Peng Zeng, ${ }^{1,3}$ Min Li, ${ }^{2,3}$ Qiong Zhang, ${ }^{3}$ Xing Li, ${ }^{1,3}$ Quanle Xu, ${ }^{1}$ Tao Li, ${ }^{4}$ Xiaojie Wang, ${ }^{2,3}$ Zhensheng Kang, ${ }^{2,3,}$ and Xinmei Zhang ${ }^{1,3, *}$

1 College of Life Sciences, Northwest A\&F University, Yangling 712100, Shaanxi, China

2 College of Plant Protection, Northwest A\&F University, Yangling 712100, Shaanxi, China

3 State Key Laboratory of Crop Stress Biology for Arid Areas, Northwest A\&F University, Yangling 712100, Shaanxi, China

4 Jiangsu Key Laboratory of Crop Genomics and Molecular Breeding, Agricultural College of Yangzhou University, Yangzhou 225009, Jiangsu, China

* Authors for correspondence:

E-mail: kangzs@nwsuaf.edu.cn; xinmeizhang@nwsuaf.edu.cn

One-sentence summary: $T a B \ln 1$ negatively regulate wheat resistance to stripe rust possibly due to the interaction with $\mathrm{TaCaM} 3$ on the plasma membrane, which impairs the calcium influx modulated by TaCaM3.

\section{List of authors' contributions}

S.Y.G., Z.S.K., X.J.W., and X.M.Z. conceived and designed the experiments; Q.Z., Q.L.X., T.L. contributed to the original concept of the project. S.Y.G., Y.Q.Z., P.Z., M.L., X.L. performed the experiments; S.Y.G. and X.L. performed the data analyses. S.Y.G. wrote the manuscript. Z.S.K., X.J.W., and X.M.Z. revised the manuscript.

\section{Funding information}

This work was supported by the Shaanxi Innovation Team Project (grant no. 2018TD-004), the 111 Project of the Ministry of Education of China (grant no. B07049), the College Student Innovation and Entrepreneurship Training Program (grant no. S202010712115) and the open funds of the Jiangsu Key Laboratory of Crop Genomics and Molecular Breeding (grant no. PL202001). 


\section{Abstract}

37 Blufensin1 $(\mathrm{B} \ln 1)$ has been identified as a negative regulator of basal defense mechanisms that is unique to the cereal grain crops barley, wheat, and rice. However, the molecular mechanisms through which Blufensinl regulates the wheat immune response are poorly understood. In this study, we found that TaBlnl is significantly induced by Puccinia striiformis f. sp. tritici (Pst) virulent race CYR31 infection. Knockdown the expression of $T a B \ln 1$ by virus-induced gene silencing reduced Pst growth and development, and enhanced the host defense response. In addition, TaBln1 was found to physically interact with TaCaM3 on the plasma membrane. Silencing TaCaM3 with virus-induced gene silencing increased fungal infection areas and sporulation and reduced wheat resistance to the Pst CYR23 and CYR31. Moreover, we found that the TaCaM3 transcription level could be induced by treatment with chitin but not flg22. Silencing TaCaM3 decreased the $\mathrm{Ca}^{2+}$ influx induced by chitin, but silencing $\mathrm{TaB} \ln 1$ increased the $\mathrm{Ca}^{2+}$ influx in vivo using a non-invasive micro-test technique. Taken together, we identified the wheat negative regulator TaBln1, which interacts with $\mathrm{TaCaM} 3$ to impair $\mathrm{Ca}^{2+}$ influx and inhibits plant defenses. 


\section{Introduction}

Negative regulators of plants, a type of susceptibility factor encoded by susceptibility genes ( $\mathrm{S}$ genes), are recruited and utilized by pathogens to interfere with plants' defensive responses; this directly or indirectly helps the growth and parasitism of pathogens (Pavan et al., 2010; Lapin et al., 2013; Schie et al., 2014). Altering S genes could result in a more broad-spectrum and durable natural resistance in plants (Pavan et al., 2010; Schie et al., 2014). Some successful examples are the use of mildew-resistance locus $O(M L O)$ mutations in barley, wheat, and tomato, which confer heritable resistance to powdery mildew. Loss of function in $M L O$ mutants results in durable and broad-spectrum resistance (Jørgensen, 1992; Acevedo-Garcia et al., 2015; Wang et al., 2014; Nekrasov et al., 2017). Likewise, simultaneous mutation of the three homologues of enhanced disease resistance 1 in wheat using CRISPR/Cas has enhanced resistance to powdery mildew (Zhang et al., 2017). Some researchers have generated rice lines with broad-spectrum resistance to $X$. oryzae $\mathrm{pv}$. oryzae by editing the promoter of three SWEET genes using CRISPR/Cas9 in rice (Oliva et al., 2019; Xu et al., 2019). Similarly, Pi21 encodes a proline-rich protein in which mutation through RNA interference can confer resistance to $M$. oryzae in rice (Fukuoka et al., 2009). Another example involves RACB, a member of the plant RAC/ROP family of RHO-like small monomeric G-proteins. Transient RNA interference through the double-stranded RNA (dsRNAi) of barley HvRacB induces partial $B g h$ resistance (Schultheiss et al., 2002; 2003). These results clearly show that identifying the precise role of negative regulators in plants is part of a powerful approach for generating resistance in plants.

Blufensin1 $(B \ln 1)$, which is a member of a new small peptides family, was initially identified as a negative regulator of basal defense mechanisms (Caldo et al., 2004). Computational interrogation of the $B \ln 1$ gene family determined that these peptides are uniquely encoded in the cereal grain crops barley, wheat, and rice. Suppressed expression of Blnl via barley stripe mosaic virus (BSMV)-induced gene silencing decreased susceptibility in compatible interactions between barley and powdery mildew. However, overexpression of $B \ln 1$ significantly increased accessibility to virulent Blumeria graminis f. sp. hordei (Bgh) (Meng et al., 2009). Recently, transient overexpression of $B \ln 2$ (a blufensin with high sequence similarity to $B \ln 1$ ) in barley has been found to increase the susceptibility of barley to $B g h$ as well. Interestingly, bimolecular fluorescence complementation (BiFC) assays have shown that BLN1 and BLN2 can interact with the $\mathrm{Ca}^{2+}$ sensor calmodulin (CaM) (Xu et al., 2015). However, despite these advances, it remains to be seen whether family members of BLN and $\mathrm{CaM}$ antagonize or cooperate with each other in modulating immune responses in wheat. 
In plant cells, calcium ions are a ubiquitous intracellular second messenger involved in numerous signaling pathways, particularly in relation to the triggering of immune responses (Tuteja et al., 2007; Hilleary et al., 2020). $\mathrm{Ca}^{2+}$ elevation in the cytosol is an early essential event in pathogen response signaling cascades and leads to the activation of downstream innate immune responses (Dangl et al., 1996). For example, the recognition of the pathogen results in cyclic nucleotide production and the activation of cyclic nucleotide-gated channels, which leads to downstream generation of pivotal signaling molecules. $\mathrm{CaMs}$ and CaM-like proteins (CMLs) are involved in this signaling, functioning as $\mathrm{Ca}^{2+}$ sensors and mediating the synthesis of $\mathrm{NO}$ (Ma et al., 2011). CaMs and CMLs constitute a large family of $\mathrm{Ca}^{2+}$ binding and signaling proteins in plants. Among these proteins, CaM, containing a pair of $\mathrm{Ca}^{2+}$-binding EF-hand motifs, is the most thoroughly characterized $\mathrm{Ca}^{2+}$ sensor in plants (Bouché et al., 2005; Defalco et al., 2009). Plant genomes contain multiple loci that encode conserved $\mathrm{CaM}$ isoforms. For example, seven distinct genes encode four protein isoforms (CaM2/CaM3/ CaM5, CaM1/CaM4, CaM6, and CaM7) in Arabidopsis (McCormack et al., 2003). It has been reported that CaMs are involved in innate immunity in plants, which can transmit the initial signal of cytosolic $\mathrm{Ca}^{2+}$ elevation to downstream targets in signal transduction cascade (Ma et al., 2007). Pathogen infection results in induction and/or suppression of plant CaM isoforms (Garcia-Brugger et al., 2006). Manipulating plant CaM expression affects basal resistance to a range of pathogens (Heo et al., 1999; Takabatake et al., 2007). For example, the silencing of specific pathogen-induced $\mathrm{CaM}$ isoforms in tomato results in enhanced susceptibility to virulent necrotrophic bacteria and fungi (Takabatake et al., 2007). The expressions of SCaM4 and SCaM5 in transgenic tobacco and Arabidopsis lead to increased pathogenesis-related gene expression and enhanced resistance to bacterial, fungal, and viral pathogens (Zhu et al., 2010). CaM may be a key player in transducing pathogen-induced $\mathrm{Ca}^{2+}$ increase to the downstream components of defense signaling and contributing to plant defense responses.

Wheat (Triticum aestivum L.) is one of the most important dietary crops in the world. While growing, wheat undergoes continuous exposure to abiotic and biotic stresses in the natural environment. Puccinia striiformis f. sp. tritici (Pst), an obligate biotrophic pathogen, is the causal agent of wheat stripe rust and has become the largest biotic limitation to wheat production and the global food supply (Liu et al., 2016; Schwessinger, 2017). Genetic control is the most cost-effective strategy for reducing the threat of this disease (Ellis et al., 2014). Recent studies have indicated that broad-spectrum resistance can result from loss of function or a mutation of negative regulators (Qiang et al., 2021). Therefore, a better understanding of the mechanisms of this pathogen at the molecular level is of great importance to improve disease resistance in wheat. 
We identified and functionally characterized one Blufensin gene, TaBln1, and assessed its important role in wheat resistance to Pst. In addition, we demonstrated that $\mathrm{TaB} \ln 1$ interacts with one $\mathrm{CaM}, \mathrm{TaCam} 3$, which was shown to play a positive role in resistance to stripe rust in wheat. Concurrently, a noninvasive micro-test technique (NMT) and chitin-treatment experiments showed that transient silencing of TaCaM3 led to a decrease in calcium influx in wheat mesophyll cells; however, silencing $T a B \ln 1$ led to a significant increase in calcium influx. Our results demonstrate that $T a B \ln 1$ negatively regulates wheat resistance to $P$ st by affecting the balance between TaCam 3 and calcium, and TaCam 3 might be the target for TaBln 1 in the wheat response to Pst infection. Our results provide new insights that may lead to improved understanding of the roles of negative regulators in plant resistance.

\section{Results}

\section{$T a B \ln 1$ is significantly induced by the Pst virulent race CYR31}

Using a complete open reading frame, the novel gene $T a B \ln 1$ was isolated from the transcriptome of wheat cultivar Suwon 11 infected by virulent Pst CYR31. BLAST analyses found in the Ensemble Plants database revealed that three copies of TaBln1 were located on chromosomes $4 \mathrm{~A}, 5 \mathrm{~B}$, and $5 \mathrm{D}$, but no complete sequence information was found. Subsequently, the copies of $T a B \ln 1$ were obtained by genomic PCR with specific primers (Supplemental Table 1), according to the wheat genome database. The three copies shared $97.33 \%$ DNA identity and $97.56 \%$ protein identity, indicating that they may possess identical biological functions.

To investigate whether $T a B \ln 1$ participates in the wheat response to Pst, we used RT-qPCR to analyze the relative transcript profiles of $T a B \ln$ with a pair of conservative primers in the $P s t$ virulent race CYR31 and the avirulent race CYR23 in inoculated wheat at different time points. The expression of $T a B \ln 1$ was upregulated at 12,24 , and $72 \mathrm{~h}$ post inoculation (hpi) in wheat leaves challenged with the Pst virulent race CYR31 (compatible interaction). The highest $T a B \ln 1$ transcript level was approximately 9-fold at 24 hpi. However, the expression of TaBln1 showed no significant change in wheat leaves challenged with the avirulent race CYR23 (Figure 1), suggesting that $T a B \ln 1$ may participate in a compatible interaction between wheat and Pst.

\section{Silencing of $T a B \ln 1$ reduces wheat susceptibility to Pst}

To determine the role of $T a B \ln 1$ in the wheat-Pst interaction, we used a BSMV-induced gene silencing (VIGS) system to knock down the expression of TaBln1. Photobleaching was observed in plants inoculated with BSMV:TaPDS, and mild chlorotic mosaic symptoms were displayed in other BSMV-infected plants with no evident defects (Figure 2A). BSMV:TaPDS was used as a positive control to indicate the efficacy of VIGS system. Thereafter, the Pst CYR31 was used to 
167

168

169

170

171

172

173

174

175

176

177

178

179

180

181

182

183

184

185

186

187

188

189

190

191

192

193

194

195

196

197

198

199

200

201

202

203

204

205

inoculate the BSMV-treated plants. The disease phenotype showed that there were fewer uredinia on TaBln1-knockdown plants at 14 days post infection (dpi) than on the control plants (Figure 2B). Moreover, the fungal biomass was significantly lower on TaBln1-knockdown plants at 5, 7, and 10 dpi than on the control plants, which is consistent with the disease phenotype (Figure 2C). Standard curves (Supplemental Figure S2) for biomass were generated using qPCR with total genomic DNA (Yin et al., 2009). The transcriptional levels of TaBln1 in BSMV:TaBln1-inoculated leaves were about 40-95\% lower at 0, 24, 48, and 120 hpi than in control plants (Figure 2D), indicating that the expression of TaBlnl was substantially knocked down by VIGS.

Knockdown of TaBln1 expression reduces Pst growth and development and enhances the host defense response

To quantify the reduced disease phenotype in TaBlnl-knockdown plants, we also microscopically examined fungal growth and development in TaBln1-silenced leaves (Figure 3A). The number of hyphal branches and haustoria at $24 \mathrm{hpi}$ and the length of hyphae at 48 hpi were significantly reduced in $T a B \ln 1$-silenced plants relative to control plants inoculated with BSMV: $\gamma$ (Figure 3B and C). Compared to the control plants, the infection areas of Pst at $120 \mathrm{hpi}$ also decreased by silencing of TaBln 1 (Figure 3D). Moreover, to further analyze the host response, we measured the accumulation of $\mathrm{H}_{2} \mathrm{O}_{2}$ in TaBlnl-knockdown plants with DAB staining. Our results revealed that the area that contained $\mathrm{H}_{2} \mathrm{O}_{2}$ was significantly larger in $T a B \ln 1$-silenced leaves than in the control leaves at 24, 48, and 120 hpi (Supplemental Figure S3). These histological results indicate that the silencing of $T a B \ln 1$ restricted the growth and development of $P s t$ and enhanced the resistance of wheat to Pst.

\section{TaBln1 interacts with the TaCaM3}

BLN1 and BLN2 were found to interact with a CaM in barley (Xu et al., 2015), suggesting that $\mathrm{TaB} \ln 1$ may interact with a $\mathrm{CaM}$ in wheat. To confirm this prediction, TaCaM3, a homologue of barley CaM, was cloned in wheat cultivar Suwan11. BIFC was first used to detect whether TaBln1 interacts with TaCaM3 in transiently transformed $N$. benthamiana leaves. Strong fluorescence signals were obtained in the interaction between TaBln1 and TaCaM3 after 48 hpi (Figure 4A). However, there were no fluorescence signals for the co-expression of NE-TaBln1 and the empty vector CE in $N$. benthamiana leaves (Figure 4A). To further investigate their physical association with co-immunoprecipitation (Co-IP) assays, we transiently co-expressed TaBln1 fused with the GFP tag and TaCaM3 fused with the HA tag in N. benthamiana. As expected, TaCaM3 was co-immunoprecipitated with TaBln1 but not with GFP (Figure 4B). Next, their interaction was further confirmed in split-luciferase assays. The nLUC fusion of TaBln1 and cLUC fusion of TaCaM3 were co-expressed in $N$. benthamiana. Only the area co-expressing TaBln1-nLUC and TaCaM3-cLUC had a strong luminescence signal; no luminescence signals appeared in the area 
co-expressing TaBLN1-nLUC and cLUC, nLUC and TaCaM3-cLUC, or nLUC and cLUC (Figure 4C). In addition, the in vitro interaction between TaBln1 and TaCaM3 was tested using a pulldown assay with TaBln1 fused with GST tag and TaCaM3 fused with His tag. When detected with anti-His monoclonal antibodies, TaCaM3 was detected in Western blots of proteins eluted from TaBln1-GST beads, but no TaCaM3 was detected from free GST beads (Figure 4D). These data confirmed that TaBln1 interacts with TaCaM3.

\section{TaCaM3 co-localizes with TaBln1 to the plasma membrane}

To determine the subcellular localization of TaBln1, we first analyzed the protein sequence for TaBln1. TaBln1 contained a signal peptide (1-29 aa) and a putative transmembrane domain (Supplemental Figure S4), indicating that it might be localized to the biological membrane. To confirm this prediction, TaBln1-GFP and a marker protein of plasma membrane, TaWpi6-mCherry, were co-expressed in $N$. benthamiana leaves. As expected, GFP fluorescent signals of TaBln1-GFP were detected in the plasma membrane and nuclear membrane; meanwhile, the green fluorescence substantially overlapped with the red fluorescence of TaWpi6-mCherry (Figure 5A). To further validate the localization of TaBln1, plasmolysis was induced by the addition of $800 \mathrm{mM}$ mannitol, and then, clear plasma membrane and nuclear membrane signals were observed for TaBln1-GFP (Figure 5B), indicating that TaBln1 was localized to the plasma membrane and nuclear membrane. In addition, in view of the interaction between TaBln1 and TaCaM3, we used TaCaM3-GFP and TaBln1-mCherry to determine whether TaBln1 co-localized with TaCaM3 to the plasma membrane and nuclear membrane. We determined the subcellular localization of TaCaM3, which, similar to the free GFP, was detected throughout the cytosol, plasma membrane, and nucleus (Supplemental Figure S5). However, the results of co-localization showed that the red fluorescence of TaBln1-mCherry substantially overlapped with the green fluorescence of TaCaM3-GFP in the plasma membrane but not with the nuclear membrane (Figure 5C). This suggests that TaBln1 mainly co-localized with TaCaM3 to the plasma membrane.

\section{Silencing of $\mathrm{TaCaM3}$ reduces wheat resistance to Pst}

To obtain direct evidence for the function of $\mathrm{TaCaM3}$, we first assayed the expression of TaCaM3 at different levels of Pst infection. The six copies of TaCaM3 on chromosomes 2A, 2B, 2D, 4A, 4B, and 4D share 95.5\% similarity in nucleotide sequence (Supplemental Figure S6) and nearly 100\% similarity in amino acid sequence (Supplemental Figure S7), which led to the design of conservative TaCaM3 primers for RT-qPCR. The transcription of TaCaM3 was significantly induced in both compatible and incompatible interactions at 12 and 24 hpi. The highest TaCaM3 transcript level was approximately 3-fold in incompatible interaction at 48 hpi (Supplemental Figure S8), suggesting that TaCaM3 plays a role in the interaction 
between wheat and Pst.

To determine the function of TaCaM3 in the wheat-Pst interaction, we silenced TaCaM3 using VIGS. When TaCaM3-knockdown plants were inoculated with CYR23, conspicuous HR was elicited on leaves that had previously been infected with BSMV: $\gamma$ and BSMV:TaCaM3 (Figure 6A). However, a slight sporulation of Pst emerged around the necrotic regions on leaves infected with BSMV:TaCaM3 at 14 dpi (Figure 6A). Although there was no obvious difference between the BSMV:TaCaM3 and BSMV: $\gamma$ plants in fungal biomass at $10 \mathrm{dpi}$, the fungal biomass was significantly increased in BSMV:TaCaM3 leaves at 5 and 7 dpi, relative to control plants (Figure 6B). Relative to leaves inoculated with BSMV: $\gamma$, the transcription of the endogenous TaCaM3 was successfully silenced in the fourth leaves of BSMV:TaCaM3 plants (Figure 6C). Moreover, we microscopically examined TaCaM3-silenced leaves infected with Pst CYR23. The length of the hyphae and the numbers of hyphal branches, haustorial mother cells, and haustoria at 24 hpi were significantly greater in the TaCaM3-silenced plants than the control plants (Figures 6D and E). At 120 hpi, the infection areas of Pst in TaCaM3-silenced plants were much larger than those in the control plants (Figure 6F). In addition, to study the effects of the knockdown of TaCaM3 on the expression of defense-related genes in wheat seedling leaves inoculated with CYR23, we assayed the expression of three PR genes in TaCaM3-silenced plants. The transcriptions of TaPR1, TaPR2, and TaPR5 were reduced at 24 and $48 \mathrm{hpi}$ in the TaCaM3-knockdown plants (Figure 6G), indicating that silencing of TaCaM3 may reduce the resistance of wheat.

Because of the high transcriptional levels of TaCaM3 in compatible interactions, we also used the CYR31 to inoculate TaCaM3-knockdown plants. Although all leaves inoculated with CYR31 produced numerous uredospores (Figure 7A), the fungal biomass was significantly increased in BSMV:TaCaM3 leaves at 5 dpi compared to the control plants (Figure 7B). The silencing efficiency analyses performed by RT-qPCR indicated that TaCaM3 was significantly knocked down in leaves inoculated with CYR31 at 0, 24, 48, and 120 hpi (Supplemental Figure S9). Moreover, histological observations showed that the hyphal length at 24 hpi (Figure 7C) and infection areas of Pst at 120 hpi (Figure 7D) were also greater after the silencing of TaCaM3 than plants inoculated with BSMV: $\gamma$. These results also confirmed that silencing of TaCaM3 reduced the wheat resistance to Pst, and TaCaM3 may contribute to the basal immune response to Pst in wheat.

\section{TaBIn1 impairs $\mathrm{Ca}^{2+}$ influx possibly by interacting with TaCaM3}

CaMs, as a universal $\mathrm{Ca}^{2+}$ sensor, can transmit the initial signal of cytosolic $\mathrm{Ca}^{2+}$ elevation (upon pathogen perception) to downstream targets in a signal transduction cascade (Garcia-Brugger et al., 2006). In this study, to further study the function of TaCaM3, two PAMPs (chitin and flg22) treatments were selected to measure the 
TaCaM3 transcript levels by RT-qPCR. The results of RT-qPCR showed that TaCaM3 was only upregulated upon chitin treatment (Figure 8A) but did not change upon flg22 treatment (Figure 8B). This indicated that chitin treatment could be used for further study.

We also investigated whether TaCaM3 may affect $\mathrm{Ca}^{2+}$ influx by measuring the dynamics of $\mathrm{Ca}^{2+}$ flux in mesophyll cells after treatment with chitin in vivo, using a NMT. All of the leaves tested were clearly responsive to chitin treatment. In response to chitin stimulation, the mesophyll cells of control plants inoculated with BSMV: $\gamma$ exhibited robust $\mathrm{Ca}^{2+}$ influx; however, little change was seen in TaCaM3 knockdown plants (Figure 9A). Because TaBln1 can interact with TaCaM3, we speculated that TaBln1 might also affect the $\mathrm{Ca}^{2+}$ influx by influencing TaCaM3. To test this hypothesis, we also measured the $\mathrm{Ca}^{2+}$ influx in TaBln1 knockdown plants. The results showed that the mesophyll cells of plants inoculated with BSMV:TaBln1, but not those in control plants, exhibited stronger and more rapid $\mathrm{Ca}^{2+}$ influx (Figure 9B). These results suggest that $\mathrm{TaCaM} 3$ could affect $\mathrm{Ca}^{2+}$ influx, and this ability was impaired by interaction with $\mathrm{TaB} \ln 1$.

\section{Discussion}

The use of loss-of-function mutations in $\mathrm{S}$ genes can save expenditure on pesticides and fungicides and eliminate the negative impacts on the environment that such approaches may have. Hence, the study of S genes is of great interest for engineering plant disease resistance. In this study, we identified $T a B \ln 1$ in wheat, which had high expression levels in the early stages of a compatible interaction but showed no significant change in an incompatible interaction. Thus, we speculated that $\operatorname{TaB} \ln 1$ may play a role in wheat susceptibility. Similar findings regarding $B l n$ genes have been reported in barley: the overexpression $B \ln 1$ and $B \ln 2$ by BSMV significantly increased susceptibility of barley to Bgh (Meng et al., 2009; Xu et al., 2015), whereas the silencing of $B \ln 1$ enhanced barley resistance in compatible interactions (Meng et al., 2009). In this study, we found that silencing TaBlnl limited the hyphal growth and fungal colony areas of Pst and increased ROS accumulation of wheat, resulting in a reduction in the number of Pst uredinia. Thus, we demonstrated that the function of the $T a B \ln 1$ gene is similar to the $B \ln 1$ and $B \ln 2$ genes of barley, which played a negative regulatory role in the interaction between wheat and Pst. Interestingly, the $B l n$ genes may be unique to the cereal grain crops barley, wheat, and rice (Meng et al., 2009). The identification of $T a B \ln 1$, as a negative regulatory factor, would provide a preliminary target in the future for editing Bln genes with CRISPR to achieve durable disease control.

Previous studies have indicated that Bln family members in barley are cysteine-rich 
small peptides (Meng et al., 2009), and both $\mathrm{B} \ln 1$ and $\mathrm{B} \ln 2$ interact with $\mathrm{CaM}(\mathrm{Xu}$ et al., 2015). In this study, we compared the amino acids of B $\ln 1$ in wheat with $B \ln 1$ and Bln2 in barley and found that they all had conserved cysteines (Supplemental Figure $\mathrm{S} 10)$. These conserved cysteines in $\mathrm{CaM}$ targets are important for $\mathrm{CaM}$-target interactions (Moore et al., 1999). Thus, we speculated that TaBln1 possessed a function somewhat similar to $\mathrm{HvB} \ln 1$ and $\mathrm{HvBln} 2$, which may interact physically with CaM. Interestingly, our results of BiFC, pulldown, LUC, and Co-IP analyses are in support of this speculation. This interaction between TaBln1 and TaCaM3 implies that they might be regulated differently during wheat $-P s t$ interactions. We speculate that $T a B \ln 1$ negatively regulates immunity in wheat by affecting the function of TaCaM3.

CaMs play a critical role in plant defense (Takabatake et al., 2007; Zhu et al., 2010). $\mathrm{TaCaM} 3$, a CaM, is a highly conserved intracellular calcium sensor (Bouché et al., 2005; Defalco et al., 2009). In this study, we identified TaCaM3 as a positive regulator of basal immunity against the $P s t$ fungus, and we found that transiently silencing TaCaM3 in wheat decreased resistance to fungal infection, which increased areas of infection and sporulation. Numerous studies have suggested a key role for CaM-mediated calcium signaling in plant growth and stress responses (Liu et al., 2003; Du et al., 2009). Thus, we speculated that TaCaM3 plays a positive regulatory role in the interaction between wheat and Pst through TaCaM3-mediated calcium signaling. To test this hypothesis, we chose chitin from two PAMPs (chitin and flg22) as a great substitute for $P s t$, which has certain limitations, for further study.

Intracellular calcium transients during plant-pathogen interactions are necessary early events that lead to local and systemic acquired resistance (Lecourieux et al., 2006). Calcium binding to CaMs regulates cellular processes directly by binding to specific DNA sequences and modulates gene expression indirectly by interacting with other proteins in a $\mathrm{Ca}^{2+}$-dependent manner and modulating their activity (Reddy et al., 2011). Therefore, intracellular calcium transients are essential for CaMs to function in plant defense signaling pathways. In this study, we measured the dynamics of $\mathrm{Ca}^{2+}$ flux in mesophyll cells after treatment with chitin in vivo using NMT. The results indicated that transiently silencing TaCaM3 decreased the $\mathrm{Ca}^{2+}$ influx induced by chitin, consistent with the results of VIGS experiments. We speculated that transient silencing of TaCaM3 affected the balance between calcium binding and calcium influx and ultimately weakened the disease resistance of wheat plants. Previous studies have indicated that $\mathrm{CaM}$ induces a net $\mathrm{Ca}^{2+}$ influx into protoplasts, leading to an increase in cytoplasmic $\mathrm{Ca}^{2+}$ levels (Wang et al., 2009a). In line with this finding, our results indicated that TaCaM3 affected $\mathrm{Ca}^{2+}$ influx. In addition, we proved that TaBln 1 could interact with $\mathrm{TaCaM} 3$, so we speculated that TaBln1 might also affect $\mathrm{Ca}^{2+}$ influx by affecting the function of TaCaM3. Interestingly, transiently silencing 
361

362

363

364

365

366

367

368

369

370

371

372

373

374

375

376

377

378

379

380

381

382

383

384

385

386

387

388

389

390

391

392

393

394

395

396

397

398

399

TaBlnl produced a stronger and more rapid $\mathrm{Ca}^{2+}$ influx, which was also consistent with the results of VIGS. Recently, similar findings on the relationship between $\mathrm{Ca}^{2+}$ influx and immune responses have been reported. For example, by comparing the calcium influx in the mesophyll cells of WT and transgenic rice plants after chitin or flg22 treatments, a study found that activated OsCNGC9 induced extracellular $\mathrm{Ca}^{2+}$ influx and then triggered ROS burst and PTI-related gene expressions, which ultimately led to enhanced disease resistance in rice (Wang et al., 2019). Therefore, we speculated that TaCaM3 could affect $\mathrm{Ca}^{2+}$ influx, and this ability was impaired by the interaction with $\mathrm{TaB} \ln 1$. TaCaM3 could not effectively transmit the initial signal of cytosolic $\mathrm{Ca}^{2+}$ elevation to downstream targets in a signal transduction cascade, which led to a weakening of plant disease resistance. To confirm our speculation, the relative expression of $T a C a M 3$ in $T a B \ln 1$-silenced leaves was determined by RT-qPCR. As expected, silencing of TaBln1 increased the transcription of TaCaM3 (Supplemental Figure S11).

In addition, analyses of the $T a B \ln 1$ sequence found that $T a B \ln 1$ is a three-copy gene located on chromosomes 4A, 5B, and 5D. Because T (4AL; 5AL) exists in diploid $T$. monococcum (Dubcovsky et al., 1996), the evolution of the wild emmer wheat chromosome $4 \mathrm{~A}$ must have started with this translocation. In the evolution of wheat chromosomes 4A, 5A, and 7A, wheat chromosome 4A was subjected to chromosome rearrangements a reciprocal translocation T (4AL; 5AL) (Dvorak et al., 2018). Analyses of the TaBln 1 protein structure found that although TaBln1 contains a signal peptide (1-19AA), it also has a putative transmembrane domain (Supplemental Figure S4). In Arabidopsis, receptor kinase FLS2 contains a signal peptide and a transmembrane domain, which can be targeted to the plasma membrane (Gómez-Gómez et al., 2000). OsCERK1, which encodes a receptor-like kinase containing a signal peptide, an extracellular domain, a transmembrane region, and an intracellular Ser/Thr kinase domain, is also localized to the plasma membrane (Shimizu et al., 2010). Therefore, we speculate that TaBln1 possess a similar localization to the aforementioned proteins. As expected, this study confirmed the localization of TaBln1 on the plasma membrane and nuclear membrane through tobacco subcellular localization. At the same time, a plasmolysis experiment confirmed the localization of TaBln1 (Figure 5B; Supplemental Figure S12). This result is different from the localization of BLN1 and BLN2, which can be secreted to the apoplast in barley (Xu et al., 2015). Consistent with the negative control free GFP but in contrast with the positive control SP(TaPR1a)-GFP (Bi et al., 2020), TaBln1, after plasmolysis, was still localized to the membrane, and no apoplastic signals were observed (Supplemental Figure S12). We speculated that this may be related to its negative regulatory function in the interaction between wheat and Pst. Interestingly, the results of co-localization showed that TaCaM3 and TaBln1 were co-localized to 
400

401

402

403

404

405

406

407

408

409

410

411

412

413

414

415

416

417

418

419

420

421

422

423

424

425

426

427

428

429

430

431

432

433

434

435

436

437

the plasma membrane, and the BIFC results showed that the fluorescence signal is mainly distributed at the plasma membrane. Therefore, we speculate that the interaction between TaBln1 and TaCaM3 mainly occurs at the plasma membrane. TaBln1 affects the calcium influx modulated by TaCaM3, possibly by hijacking TaCaM3 to the plasma membrane.

\section{Conclusions}

The present study reveals the potential molecular mechanism of TaBln1 in the wheat-Pst interaction. In uninoculated wheat leaves (Figure 10A), the expression of $T a B \ln 1$ is maintained at a low level. TaCaM3 functions normally in binding $\mathrm{Ca}^{2+}$, and it may be transferred to downstream targets to activate the immune responses. During Pst infection of wheat (Figure 10B), the expression of TaBln1 may be upregulated and localized to the plasma membrane. The interaction between TaBln 1 and TaCaM3 results in the accumulation of TaCaM3 on the plasma membrane. This, in turn, affects the balance between calcium binding and calcium influx, thereby reducing the transmission of $\mathrm{Ca}^{2+}$ signals to downstream targets, and ultimately weakening the disease resistance of wheat. Obviously, a better understanding of the physiological significance of the cytosolic $\mathrm{Ca}^{2+}$ changes in plant-pathogen interactions will involve the identification and functional analysis of downstream targets of cytosolic $\mathrm{Ca}^{2+}$. Thus, most intracellular target proteins that sense and relay $\mathrm{Ca}^{2+}$ signatures toward the appropriate defense responses remain possible objects of future testing.

\section{Materials and Methods}

\section{Plant materials, inoculation, and treatment}

Wheat (Triticum aestivum cv. Suwon 11), Nicotiana benthamiana, and two Pst pathotypes, CYR23 (avirulent) and CYR31 (virulent), were used in this study. Suwon 11 exhibits a high resistance to CYR23 but high susceptibility to CYR31 (Cao et al., 2003). Wheat seedling cultivation and inoculation with Pst were performed as described previously (Kang et al., 2002). Leaves were collected at 0, 12, 24, 48, 72, and 120 post-inoculation (hpi) for RNA isolation (Wang et al., 2007). The $N$. benthamiana was grown at $22^{\circ} \mathrm{C}$ and used for transient expression.

\section{Genomic DNA, total RNA extraction, and cDNA synthesis}

Genomic DNA was extracted using the cetyltrimethylammonium bromide (CTAB) method (Porebski et al., 1997). The total RNA from the wheat leaves was isolated using a Quick RNA isolation Kit (TIANGEN BIOTECH), according to the manufacturer's instructions. The extracted RNA was reversely transcribed into cDNA using the RevertAid First Strand cDNA Synthesis Kit (Thermo Scientific) and oligo $(\mathrm{dT})_{18}$ primer following the manufacturer's protocol. 


\section{Cloning of TaBln1 and TaCam3 and sequence analysis}

To clone the TaBln1 and TaCam3 sequences, specific primers (Supplementary Table S1) were designed using the Primer 5.0 software. The TaBln1 and TaCam3 sequences were PCR-amplified using Suwon 11 cDNA sample as a template. The obtained sequences were aligned using the T. aestivum cv. Chinese Spring (CS) genome from the Ensemble Plants database (http://plants.ensembl.org/Multi/Tools/Blast) and the NCBI (http://www.ncbi.nlm.nih.gov/). The multi-sequence alignments were carried out using DNAMAN 8 software. The molecular sizes of TaBln1 and TaCam3 were predicted using the Compute pI/Mw tool (https://web.expasy.org/compute_pi/). The signal peptide of $T a B \ln 1$ was identified using SignalP 5.0 (http://www.cbs.dtu.dk/services/SignalP/). The transmembrane domain of TaBln1 was identified using TMHMM Server v. 2.0 (http://www.cbs.dtu.dk/services/TMHMM/).

\section{Plasmid construction}

To construct the vector of subcellular localization, the coding sequences for TaBln1 and TaCaM3 without a stop codon were amplified and inserted into the NcoI and SpeI restriction sites of the plant binary expression vector pCAMBIA1302 containing the GFP-tag sequence, respectively. In addition, the TaBln1 without a stop codon was inserted into the pICH86988 vector with the mCherry tag.

For plasmid constructs for the silencing system, two small cDNA fragments based on the results of a BLASTN search of the NCBI (http://www.ncbi.nlm.nih.gov/) that showed the lowest sequence similarity with other wheat genes were inserted into PacI and NotI restriction sites of the virus plasmid $\gamma$, respectively (Holzberg et al., 2002).

For the interaction analyses of TaBln1 and TaCam3 using BIFC, TaBln1 and TaCam3 were subcloned into pSPYNE(R)173 and pSPYCE(M) with BamHI and XhoI restriction sites to generate the pSPYNE(R)173-TaBln1 and pSPYCE(M)-TaCam3 vectors, respectively (Waadt et al., 2008). The coding sequence of TaBln1 was inserted into the SpeI restriction site of pBinGFP2 vector and TaCam3 with HA tag was inserted into the $B s a \mathrm{I}$ restriction site of pICH86988 vector to generate pBinGFP2-TaBln1 and pICH86988-TaCam3 for co-immunoprecipitation. The coding sequences of $T a B \ln 1$ and TaCam3 were subcloned into the N- or C-terminal fragment of LUC (nLUC or cLUC) with BamHI and SalI restriction sites to generate the TaBln1-nLUC and TaCam3-cLUC vectors for split-luciferase assays, respectively. The coding sequence of TaBlnl was subcloned into the EcoRI and XhoI restriction sites of the pGEX-4T-1 expression vector containing the GST-tag sequence; the coding sequence of TaCam3 was subcloned into the EcoRI and XhoI restriction sites of the pET-28a vectors containing the His-tag sequence for the pulldown assay. The primers for all of the plasmid constructions are listed in Supplemental Table S1.

\section{BSMV-mediated gene silencing}

To transiently silence TaBln1 and TaCam3, the corresponding vectors (TaPDS- $\gamma$, 
recombinant $\gamma$-gene and $\gamma$ ), $\alpha$ and $\beta$ were linearized using corresponding enzymes and transcribed into RNA. The transcripts of $\alpha$ and $\beta$ were mixed in a 1:1:1 ratio with transcripts of TaPDS- $\gamma$, recombinant $\gamma$-gene, and $\gamma$ in FES buffer $(0.1 \mathrm{M}$ glycine $\mathrm{pH}$ 8.9, $0.06 \mathrm{M} \mathrm{K}_{2} \mathrm{HPO}_{4}, 1 \%$ bentonite, $1 \%$ sodium pyrophosphate and $1 \%$ celite), respectively. Then the second wheat leaves were inoculated with the BSMV RNA mixture and maintained at $26^{\circ} \mathrm{C}$ (Holzberg et al., 2002). BSMV: TaPDS (wheat phytoene desaturase) was used as a positive control. When the virus phenotype (photobleaching in positive control) was observed (12 days after BSMV treatment), the fourth leaves were inoculated with Pst CYR23 or CYR31 and samples were harvested at 0, 24, 48, and 120 hpi for histological observation and RNA isolation. RNA was used to confirm the silencing efficiency for each assay by RT-qPCR. The phenotypes were recorded, and representative photographs were captured at $14 \mathrm{dpi}$. Three independent sets of inoculations were performed, consisting of 60 seedlings inoculated for each BSMV virus.

\section{Histological analyses of silenced plants inoculated with Pst}

For the histological observations of fungal growth, the samples of silenced plants were cut into several fragments and decolorized in destaining solution (absolute ethyl alcohol:acetic acid, 1:1 v/v) as previously described (Cheng et al., 2015). Then these fragments were decolorized in chloral hydrate for $24 \mathrm{~h}$ followed with autoclaving at $121^{\circ} \mathrm{C}$ for 2 min. Wheat germ agglutinin (WGA) conjugated to Alexa Fluor 488 (Invitrogen) was used to stain the Pst infection structures as previously described (Ayliffe et al., 2011).

For the detection of $\mathrm{H}_{2} \mathrm{O}_{2}$ accumulation, infected leaves were cut and the ends were immersed in a solution containing $1 \mathrm{mg} / \mathrm{mL} 3,3-$ diaminobenzidine (DAB, Amresco) for 4-6 h (Wang et al., 2007). Then these fragments were decolorized in destaining solution to remove the chlorophyll. Only those infected sites where an appressorium had formed over a stoma were considered a successful penetration site of Pst. The accumulation of $\mathrm{H}_{2} \mathrm{O}_{2}$, hyphal length, infection area, and a number of hyphal branches, haustorial mother cells, and haustoria were observed with an Olympus BX-51 microscope (Olympus) and measured using the cellSens Entry software (Olympus).

\section{Protein interaction assays}

For the BiFC assay, the pSPYNE(R)173-TaBln1 and pSPYCE(M)-TaCam3 vectors were transformed into A. tumefaciens GV3101. The Agrobacterium strains were co-infiltrated at an $\mathrm{OD}_{600}$ of 0.6 into $N$. benthamiana leaves. Two days after inoculation, YFP fluorescence was captured by confocal microscopy with a $488 \mathrm{~nm}$ laser.

For the Co-IP assay, the pBinGFP2-TaBln1 and pICH86988-TaCam3 vectors were transformed into A. tumefaciens GV3101 and co-infiltrated into N. benthamiana leaves. The pBinGFP2 empty vector was used as the negative control. Two days after 
inoculation, the infiltrated leaves were harvested and ground to a powder in liquid nitrogen and then homogenized in RIPA buffer $(50 \mathrm{mM}$ Tris- $\mathrm{HCl}, \mathrm{pH} 8,150 \mathrm{mM}$ $\mathrm{NaCl}, 1 \% \mathrm{NP}-40,0.25 \%$ sodium deoxycholate, and $0.1 \%$ SDS) with $1 \mathrm{mM}$ PMSF (Beyotime Biotechnology). Then the extract was centrifuged at $12000 \mathrm{rpm}$ for $10 \mathrm{~min}$, and the supernatant was transferred into a fresh tube for the Co-IP assay. For immunodetection, the supernatant was mixed with $15 \mu \mathrm{L}$ GFP-trap A beads (Chromotek) and incubated at $4{ }^{\circ} \mathrm{C}$ for $1.5 \mathrm{~h}$. The beads were collected and washed five times with $500 \mu \mathrm{L}$ wash buffer $(50 \mathrm{mM}$ Tris- $\mathrm{HCl}, \mathrm{pH} 7.4,150 \mathrm{mM} \mathrm{NaCl}$ and $0.5 \%$ Tween-20). Proteins bound to the beads were boiled for $10 \mathrm{~min}$ and detected by Western blotting with anti-HA and anti-GFP, respectively. The monoclonal antibodies anti-HA (Beyotime Biotechnology) and anti-GFP (Beyotime Biotechnology) were used at a 1:5,000 dilution and followed by incubation with a second antibody, anti-mouse Ig-horseradish peroxidase (1:2000; Beyotime Biotechnology). Protein bands were detected using SuperSignal West Femto Maximum Sensitivity substrate (Thermo Scientific).

Split-luciferase assays were performed as previously described (Chen et al., 2008). A. tumefaciens GV3101 with different constructs were co-infiltrated into $N$. benthamiana leaves, respectively. After 2 days, $1 \mathrm{mM}$ luciferin (AbMole) was sprayed onto the inoculated leaves, and the LUC activity was captured with a PlantView100 assay system (BLT PHOTON TECHNOLOGY).

For the pulldown assay, GST fusion proteins of TaBln1 and His fusion proteins of TaCam3 were expressed in E. coli (BL21) by induction with $0.3 \mathrm{mM}$ IPTG at $16^{\circ} \mathrm{C}$ overnight. Crude proteins of TaBln1 with GST tag were purified using standard techniques with glutathione-sepharose following a previous study (Harper et al., 2001), and TaCam3 with the His tag was purified via Ni-chelating affinity chromatography. Equal amounts of GST-tagged TaBln1 or GST (negative control) were mixed with TaCam3-His and incubated at $4{ }^{\circ} \mathrm{C}$ for $2 \mathrm{~h}$ with GST beads (Thermo Scientific). The beads were collected and washed five times using the GST Protein Interaction Pull down Kit (Thermo Scientific) according to the manufacturer's instructions to detect the recovered TaCam3-His levels. The proteins bound to the beads were boiled for $10 \mathrm{~min}$ and detected by Western blotting with anti-GST and anti-His antibodies. Monoclonal anti-GST (Beyotime Biotechnology) and anti-His (Beyotime Biotechnology) antibodies were used at a 1:5,000 dilution and followed by incubation with a second antibody, anti-mouse Ig-horseradish peroxidase (Beyotime Biotechnology). The protein bands were detected using SuperSignal West Femto Maximum Sensitivity substrate (Thermo Scientific).

\section{Subcellular localization of TaBln1 and TaCam3 in N. benthamiana}

To determine the subcellular localization of TaBln1 and TaCam3, Agrobacterium strains GV3101 harboring TaBln1-GFP and TaWpi6-mCherry, a positive control 
555

556

557

558

559

560

561

562

563

564

565

566

567

568

569

570

571

572

573

574

575

576

577

578

579

580

581

582

583

584

585

586

587

588

589

590

591

592

593

marker protein that is localized to the plasma membrane (Imai et al., 2005), were co-infiltrated into 4-week-old $N$. benthamiana leaves. To determine the co-localization of TaBln 1 and TaCam3, N. benthamiana leaves were infiltrated with GV3101 carrying TaBln1-mCherry and TaCam3-GFP. The infiltrated seedlings were transferred to a growth chamber at $22^{\circ} \mathrm{C}$ with a $16 \mathrm{~h}$ light photoperiod. GFP fluorescence signals, with an excitation laser at $488 \mathrm{~nm}$ and mCherry fluorescence signals with an excitation laser at $546 \mathrm{~nm}$ were monitored at $48 \mathrm{~h}$ post transformation using an Olympus FV3000 Confocal Laser Microscope. All of the assays were performed in duplicate and repeated at least three times.

\section{RT-qPCR analyses}

Transcription levels were analyzed using a Real-Time PCR Detection System (Bio-Rad). Primer designs and RT-qPCR reactions were conducted as described previously (Wang et al., 2009b). The wheat elongation factor TaEF-1a gene was used as a wheat internal reference. Fungal biomass changes in the Pst-infected wheat leaves were analyzed as described previously (Chang et al., 2017). Biomass was determined by absolute quantification using a double-standard curve (Lee et al., 2008). The constitutively expressed wheat stripe rust elongation factor gene PstEF1 was used for the quantification of wheat stripe rust fungus (Yin et al., 2009). All of the primers used are listed in Supplemental Table S1. RT-qPCR analyses used data from three biological repeats, with each group containing three technical repeats. Relative expression was estimated using the $2^{-\Delta \Delta \mathrm{CT}}$ method (Livak et al., 2001).

Quantification of PAMP-induced gene expression and measurements of net $\mathrm{Ca}^{2+}$ flux

The detection of PAMP-induced gene expression was conducted as previously described, with minor modifications (Park et al., 2012; Schoonbeek et al., 2015). Four $2 \mathrm{~cm}$ long strips of wheat leaves were cut and placed into $2 \mathrm{~mL}$ tubes with water and preinfiltrated by vacuum for $3 \times 1 \mathrm{~min}$. Then the materials were left to recover in a growth cabinet overnight to recover from wounding stress and water infiltration. Afterward, water was replaced by fresh water (mock) or $10 \mu \mathrm{M}$ flg22 peptide (Bankpeptide) or $10 \mu \mathrm{M}$ chitin (Santa Cruz. Biotechnology) for 10, 30, 60, or $180 \mathrm{~min}$, followed by freezing in liquid nitrogen. Materials were used for RT-qPCR assays with the primers listed in Supplemental Table S1.

Net $\mathrm{Ca}^{2+}$ flux was measured using the NMT-YG-100 (YoungerUSA) as previously described (Ma et al., 2015; Wang et al., 2019). In brief, leaves sampled from transiently silenced seedling were immobilized in measuring buffer $(0.1 \mathrm{mM} \mathrm{KCl}, 0.1$ $\mathrm{mM} \mathrm{CaCl}_{2}, 0.1 \mathrm{mM} \mathrm{MgCl} 2,0.5 \mathrm{mM} \mathrm{NaCl}, 0.3 \mathrm{mM} \mathrm{MES}, 0.2 \mathrm{mM} \mathrm{Na}_{2} \mathrm{SO}_{4}$, and $\mathrm{pH}$ 6.0) for $30 \mathrm{~min}$ equilibration. The steady-state fluxes in leaf mesophyll cells were continuously recorded for $5 \mathrm{~min}$ prior to the chitin treatments. Thereafter, the chitin was slowly added to the measuring buffer until the chitin concentration reached 10 
$594 \mu \mathrm{M}$. Then the transient flux of $\mathrm{Ca}^{2+}$ was recorded and continued for $10 \mathrm{~min}$.

595 Data analyses

596 The data were analyzed using Student's $t$-test, with GraphPad Prism 8.0 statistical

597 software to determine the significant differences between control and treatment.

598 Accession numbers

599 Sequence data from this article can be found in the National Center for Biotechnology

600 database (http://www.ncbi.nlm.nih.gov/) and the Ensembl Plants portal

601 (http://plants.ensembl.org/Triticum_aestivum/Info/Index) under the following

602 accession numbers: $T a B \ln 1-4 A$ (AK333112.1), TaBln1-5B (xxx), TaBln1-5D (xxx),

603 TaCaM3-2A (TraesCS2A02G098100), TaCaM3-2B (TraesCS2B02G113800),

604 TaCaM3-2D (TraesCS2D02G097500), TaCaM3-4A (TraesCS4A02G126700),

605 TaCaM3-4B (TraesCS4B02G178200), TaCaM3-4D (TraesCS4D02G179800),

606 TaEF-1 $\alpha$ (Q03033). TaPR1 (AF384143), TaPR2 (DQ090946), TaPR5, (FG618781).

607

608

609

610

611

612

613

614

615

616

617

618

619

620

621

622

623

624

625

626

627

628

629

630

631 


\section{References}

Acevedo-Garcia J, Kusch S, Panstruga R (2015) Magical mystery tour: MLO proteins in plant immunity and beyond. New Phytol 204: 273-281

Ayliffe M, Devilla R, Mago R, White R, Talbot M, Pryor A, Leung H (2011) Nonhost resistance of rice to rust pathogens. Mol Plant Microbe Interact 24: 1143-1155

Bi W, Zhao S, Zhao J, Su J, Yu X, Liu D, Kang Z, Wang X, Wang X (2020) Rust effector PNPi interacting with wheat TaPR1a attenuates plant defense response. Phytopathology Res 2: 1-14

Bouché N, Yellin A, Snedden WA, Fromm H (2005) Plant-specific calmodulin-binding proteins. Annu Rev Plant Biol 56: 435-466

Caldo RA, Nettleton D, Wise RP (2004) Interaction-dependent gene expression in Mla-specified response to barley powdery mildew. Plant Cell 16: 2514-2528

Cao Z, Jing J, Wang M, Shang H, Li Z (2003) Relation analysis of stripe rust resistance gene in wheat important cultivar suwon 11, suwon 92 and hybrid 46. Acta Botanica Boreali-Occidentalia Sinica 23: 64-68

Chang Q, Liu J, Lin X, Hu S, Yang Y, Li D, Chen L, Huai B, Huang L, Voegele RT, et al. (2017) A unique invertase is important for sugar absorption of an obligate biotrophic pathogen during infection. New Phytol 215: 1548-1561

Chen H, Zou Y, Shang Y, Lin H, Wang Y, Cai R, Tang X, Zhou J-M (2008) Firefly luciferase complementation imaging assay for protein-protein interactions in plants. Plant Physiol 146: 368-376

Cheng Y, Wang X, Yao J, Voegele RT, Zhang Y, Wang W, Huang L, Kang Z (2015) Characterization of protein kinase PsSRPKL, a novel pathogenicity factor in the wheat stripe rust fungus. Environ Microbiol 17: 2601-2617

Dangl JL, Dietrich RA, Richberg MH (1996) Death don't have no mercy cell death programs in plant-microbe interactions. Plant Cell 8: 1793-1807

Defalco TA, Bender KW, Snedden WA (2009) Breaking the code: $\mathrm{Ca}^{2+}$ sensors in plant signalling. Biochem J 425: 27-40

Du L, Ali GS, Simons KA, Hou J, Yang T, Reddy ASN, Poovaiah BW (2009) $\mathrm{Ca}^{2+} /$ calmodulin regulates salicylic-acid-mediated plant immunity. Nature 457: 1154-1158

Dvorak J, Wang L, Zhu T, Jorgensen CM, Luo MC, Deal KR, Gu YQ, Gill BS, Distelfeld A, Devos KM, et al. (2018) Reassessment of the evolution of wheat chromosomes 4A, 5A, and 7B. Theor Appl Genet 131: 2451-2462

Ellis JG, Lagudah ES, Spielmeyer W, Dodds PN (2014) The past, present and future of breeding rust resistant wheat. Front Plant Sci 5: 1-13

Fukuoka S, Saka N, Koga H, Ono K, Shimizu T, Ebana K, Hayashi N, Takahashi 
A, Hirochika H, Okuno K, et al. (2009) Loss of function of a proline-containing protein confers durable disease resistance in rice. Science 325: 998-1001

\section{Garcia-Brugger A, Lamotte O, Vandelle E, Bourque S, Lecourieux D, Poinssot B,} Wendehenne D, Pugin A (2006) Early signalingevents induced by elicitors of plant defenses. Mol Plant Microbe Interact 19: 711-724

Gómez-Gómez L, Boller T (2000) FLS2: an LRR receptor-like kinase involved in the perception of the bacterial elicitor flagellin in Arabidopsis. Mol Cell 5: 1003-1011 Harper S, Speicher DW (2001) Expression and purification of gst fusion proteins. Curr Protoc Protein Sci Chapter 6: Unit 6.6

\section{Heo WD, Lee SH, Kim MC, Kim JC, Chung WS, Chun HJ, Lee KJ, Park CY,} Park HC, Choi JY, et al. (1999) Involvement of specific calmodulin isoforms in salicylic acidindependent activation of plant disease resistance responses. Proc Natl Acad Sci USA 96: 766-771

Hilleary R, Paez-Valencia J, Vens C, Toyota M, Palmgren M, Gilroy S (2020) Tonoplast-localized $\mathrm{Ca}^{2+}$ pumps regulate $\mathrm{Ca}^{2+}$ signals during pattern-triggered immunity in Arabidopsis thaliana. Proc Natl Acad Sci USA 117: 18849-18857

Holzberg S, Brosio P, Gross C, Pogue GP (2002) Barley stripe mosaic virus-induced gene silencing in a monocot plant. Plant J 30: 315-327

Imai R, Koike M, Sutoh K, Kawakami A, Torada A, Oono K (2005) Molecular characterization of a cold-induced plasma membrane protein gene from wheat. Mol Genet Genomics 274: 445-453

Jørgensen JH (1992) Discovery, characterization and exploitation of Mlo powdery mildew resistance in barley. Euphytica 63: 152

Kang Z, Huang L, Buchenauer H (2002) Ultrastructural changes and localization of lignin and callose in compatible and incompatible interactions between wheat and Puccinia striiformis. J Plant Dis Protect 109: 25-37

Lapin D, Ackerveken GVD (2013) Susceptibility to plant disease: more than a failure of host immunity. Trends Plant Sci 18: 546-554

Lecourieux D, Ranjeva R, Pugin A (2006) Calcium in plant defence-signalling pathways. New Phytol 171: 249-269

Lee C, Lee S, Shin SG, Hwang S (2008) Real-time PCR determination of rRNA gene copy number: absolute and relative quantification assays with Escherichia coli. Appl Microbiol Biotechnol 78: 371-376

Liu C, Pedersen C, Schultz-Larsen T, Aguilar GB, Madriz-Ordenana K, Hovmøller MS, Thordal-Christensen H (2016) The stripe rust fungal effector PEC6 suppresses pattern-triggered immunity in a host species-independent manner and interacts with adenosine kinases. New Phytol

Liu H, Li B, Shang Z, Li X, Mu R, Sun D, Zhou R (2003) Calmodulin is involved in heat shock signal transduction in wheat. Plant Physiol 132: 1186-1195 
Livak KJ, Schmittgen TD (2001) Analysis of relative gene expression data using real-time quantitative PCR and the $2^{-\Delta \Delta C T}$ Method. Methods 25: 402-408

Ma W, Berkowitz GA (2007) The grateful dead: calcium and cell death in plant innate immunity. Cell Microbiol 9: 2571-2585

Ma W, Berkowitz GA (2011) $\mathrm{Ca}^{2+}$ conduction by plant cyclic nucleotide gated channels and associated signaling components in pathogen defense signal transduction cascades. New Phytol 190: 566-572

Ma Y, Dai X, Xu Y, Luo W, Zheng X, Zeng D, Pan Y, Lin X, Liu H, Zhang D, et al. (2015) COLDl confers chilling tolerance in rice. Cell 160: 1209-1221

Mccormack E, Braam J (2003) Calmodulins and related potential calcium sensors of Arabidopsis. New Phytol 159: 585-598

Meng Y, Moscou MJ, Wise RP (2009) Blufensin1 negatively impacts basal defense in response to barley powdery mildew. Plant Physiol 149: 271-285

Moore CP, Zhang J, Hamilton SL (1999) A role for cysteine 3635 of RYR1 in redox modulation and calmodulin binding. J Biol Chem 274: 36831-36834

Nekrasov V, Wang C, Win J, Lanz C, Weigel D, Kamoun S (2017) Rapid generation of a transgene-free powdery mildew resistant tomato by genome deletion. Sci Rep 7: 482

Oliva R, Ji C, Atienza-Grande G, Huguet-Tapia JC, Perez-Quintero A, Li T, Eom J-S, Li C, Nguyen H, Liu B, et al. (2019) Broad-spectrum resistance to bacterial blight in rice using genome editing. Nat Biotechnol 37: 1344-1350

Park CH, Chen S, Shirsekar G, Zhou B, Khang CH, Songkumarn P, Afzal AJ, Ning Y, Wang R, Bellizzi M, et al. (2012) The Magnaporthe oryzae effector AvrPiz-t targets the RING E3 ubiquitin ligase APIP6 to suppress pathogen-associated molecular pattern-triggered immunity in rice. Plant Cell 24: 4748-4762

Pavan S, Jacobsen E, Visser RG, Bai Y (2010) Loss of susceptibility as a novel breeding strategy for durable and broad-spectrum resistance. Mol Breed 25: 1-12

Porebski S, Bailey LG, Baum BR (1997) Modification of a CTAB DNA extraction protocol for plants containing high polysaccharide and polyphenol components. Plant Mol Biol Report 15: 8-15

Qiang X, Liu X, Wang X, Zheng Q, Kang L, Gao X, Wei Y, Wu W, Zhao H, Shan W (2021) Susceptibility factor RTP1 negatively regulates Phytophthora parasitica resistance via modulating UPR regulators bZIP60 and bZIP28. Plant Physiol

Reddy ASN, Ali GS, Celesnik H, Day IS (2011) Coping with stresses: roles of calcium- and calcium/calmodulin-regulated gene expression. Plant Cell 23: 2010-2032

Schie CCNV, Takken FLW (2014) Susceptibility genes 101: how to be a good host. Annu Rev Phytopathol 52: 551-581

Schoonbeek HJ, Wang HH, Stefanato FL, Craze M, Bowden S, Wallington E, 
Zipfel C, Ridout CJ (2015) Arabidopsis EF-Tu receptor enhances bacterial disease resistance in transgenic wheat. New Phytol 206: 606-613

Schultheiss H, Dechert C, Kogel K-H, Hückelhoven R (2002) A small GTP-binding host protein is required for entry of powdery mildew fungus into epidermal cells of barley. Plant Physiol 128: 1447-1454

Schultheiss H, Dechert C, Kogel K-H, Hückelhoven R (2003) Functional analysis of barley RAC/ROP G-protein family members in susceptibility to the powdery mildew fungus. Plant J 36: 589-601

Schwessinger B (2017) Fundamental wheat stripe rust research in the 21st century. New Phytol 213: 1625-1631

\section{Shimizu T, Nakano T, Takamizawa D, Desaki Y, Ishii-Minami N, Nishizawa Y,} Minami E, Okada K, Yamane H, Kaku H, et al. (2010) Two LysM receptor molecules, CEBiP and OsCERK1, cooperatively regulate chitin elicitor signaling in rice. Plant J 64: 204-214

Takabatake R, Karita E, Seo S, Mitsuhara I, Kuchitsu K, Ohashi Y (2007) Pathogen-induced calmodulin isoforms in basal resistance against bacterial and fungal pathogens in tobacco. Plant Cell Physiol 48: 414-423

Tuteja N, Mahajan S (2007) Calcium signaling network in plants. Plant Signal Behav 2: 79-85

Waadt R, Schmidt LK, Lohse M, Hashimoto K, Bock R, Kudla J (2008) Multicolor bimolecular fluorescence complementation reveals simultaneous formation of alternative CBL/CIPK complexes in planta. Plant J 56: 505-516

Wang C, Huang L, Buchenauer H, Han Q, Zhang H, Kang Z (2007) Histochemical studies on the accumulation of reactive oxygen species $\left(\mathrm{O}^{2-}\right.$ and $\left.\mathrm{H}_{2} \mathrm{O}_{2}\right)$ in the incompatible and compatible interaction of wheat - Puccinia striiformis $\mathrm{f}$. $\mathrm{sp}$ tritici. Physiol Mol Plant Pathol 71: 230-239

Wang J, Liu X, Zhang A, Ren Y, Wu F, Wang G, Xu Y, Lei C, Zhu S, Pan T, et al. (2019) A cyclic nucleotide-gated channel mediates cytoplasmic calcium elevation and disease resistance in rice. Cell Res 29: $820-831$

Wang Q, Chen B, Liu P, Zheng M, Wang Y, Cui S, Sun D, Fang X, Liu C, Lucas WJ, et al. (2009a) Calmodulin binds to extracellular sites on the plasma membrane of plant cells and elicits a rise in intracellular calcium concentration. J Biol Chem 284: 12000-12007

Wang X, Tang C, Zhang G, Li Y, Wang C, Liu B, Qu Z, Zhao J, Han Q, Huang L, et al. (2009b) cDNA-AFLP analysis reveals differential gene expression in compatible interaction of wheat challenged with Puccinia striiformis f. sp. tritici. BMC Genomics 10: 289

Wang Y, Cheng X, Shan Q, Zhang Y, Liu J, Gao C, Qiu J (2014) Simultaneous editing of three homoeoalleles in hexaploid bread wheat confers heritable resistance 
to powdery mildew. Nat Biotechnol 32: 947-951

Xu W, Meng Y, Surana P, Fuerst G, Nettleton D, Wise RP (2015) The knottin-like Blufensin family regulates genes involved in nuclear import and the secretory pathway in barley-powdery mildew interactions. Front Plant Sci 6: 409

Xu Z, Xu X, Gong Q, Li Z, Li Y, Wang S, Yang Y, Ma W, Liu L, Zhu B, et al. (2019) Engineering broad-spectrum bacterial blight resistance by simultaneously disrupting variable tale-binding elements of multiple susceptibility genes in rice. Mol Plant 12: 1434-1446

Yin C, Chen X, Wang X, Han Q, Kang Z, Hulbert SH (2009) Generation and analysis of expression sequence tags from haustoria of the wheat stripe rust fungus Puccinia striiformis f. sp. tritici. BMC Genomics 10: 626

Zhang Y, Bai Y, Wu G, Zou S, Chen Y, Gao C, Tang D (2017) Simultaneous modification of three homoeologs of TaEDRl by genome editing enhances powdery mildew resistance in wheat. Plant J 91: 714-724

\section{Zhu X, Caplan J, Mamillapalli P, Czymmek K, Dinesh-Kumar SP (2010)}

Function of endoplasmic reticulum calcium ATPase in innate immunity-mediated programmed cell death. Embo J 29: 1007-1018 


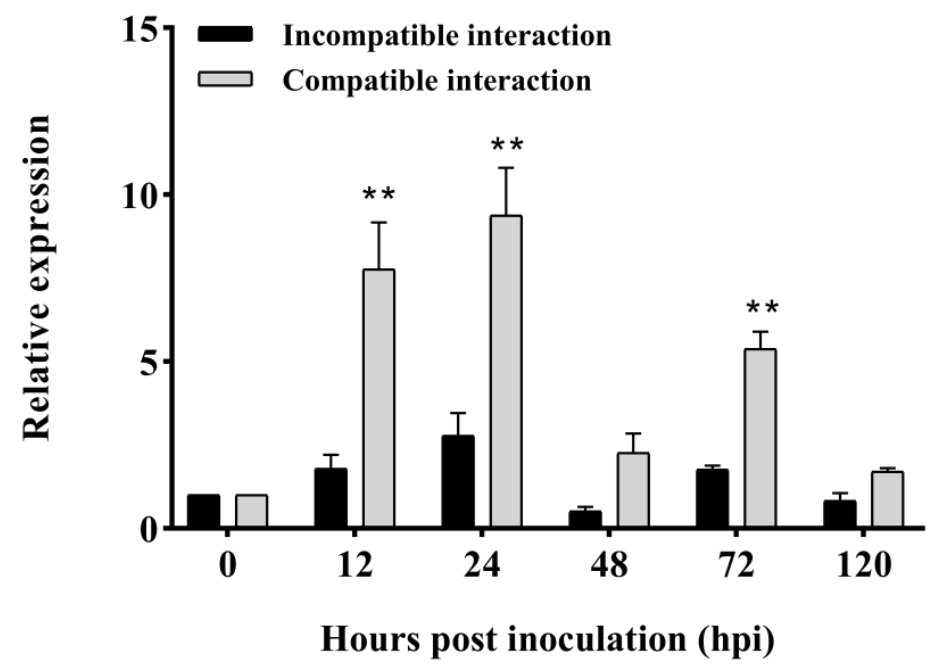

817 Figure 1. TaBln1 is significantly induced by Pst race CYR31 infection. Wheat 818 leaves were sampled for CYR23 and CYR31-inoculated plants at 0, 12, 24, 48, 72, 819 and 120 hpi. Untreated leaves act as the control. The relative expression of TaBln 1 820 was calculated using the comparative threshold method $\left(2^{-\Delta \Delta \mathrm{CT}}\right)$. Expression levels 821 were normalized to TaEF-1a. Values were derived from three biological repetitions. 822 Asterisks indicate a significant difference $(* * \mathrm{P}<0.01)$ compared to the untreated control according to Student's $t$ test. 
A

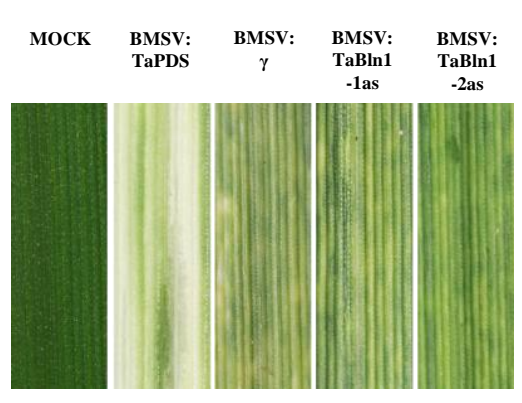

C

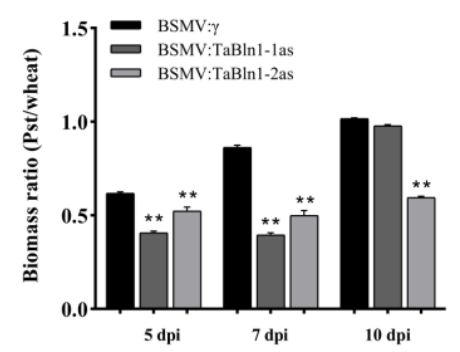

$\mathbf{B}$
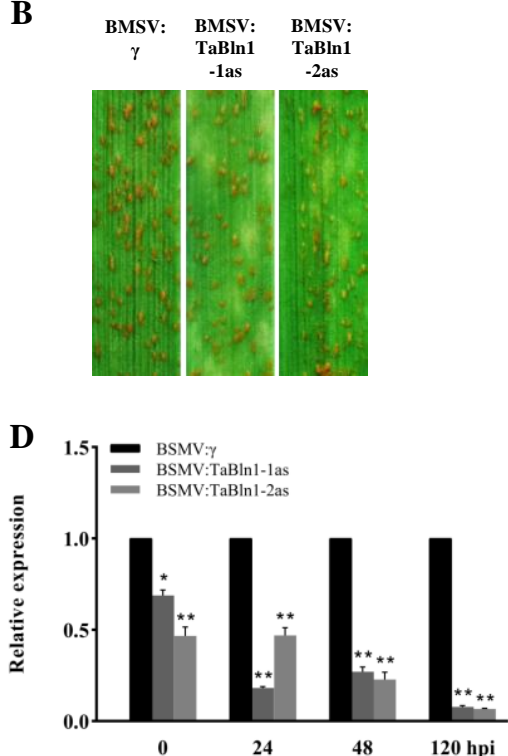

Figure 2. Silencing TaBln1 reduces wheat susceptibility to Pst race CYR31. A, The phenotype did not change in wheat leaves mock inoculated. Photobleaching was visible on wheat leaves inoculated with BSMV:TaPDS. Slight chlorotic mosaic symptoms were observed in wheat leaves infected with BSMV: $\gamma$, BSMV:TaBln1-1as and BSMV:TaBln1-2as. B, Disease phenotypes of the fourth leaves pre-inoculated with BSMV constructs and then challenged with Pst CYR31. Disease phenotypes were photographed at $14 \mathrm{dpi}$. C, Biomass ratio of the $P$ st/wheat was assayed by qRT-PCR with total DNA extracted from BSMV-treated wheat leaves infected by CYR31 at 5, 7 and 10 dpi. Ratio of total fungal DNA to total wheat DNA was assessed by normalizing the data to the wheat gene TaEF-1 $\alpha$ and the Pst gene PstEF1. D, The relative expression levels of $T a B \ln 1$ in wheat leaves inoculated with CYR31 were assayed by qRT-PCR at 0, 24, 48 and 120 hpi. 
A
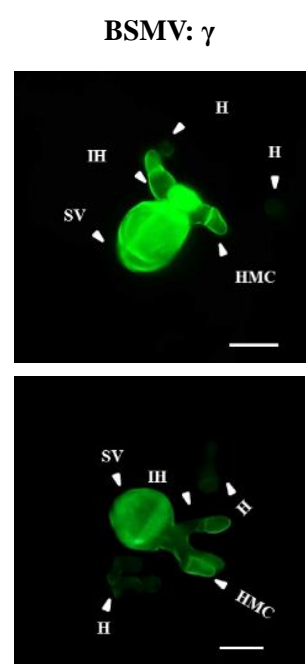

48 hpi

120 hpi

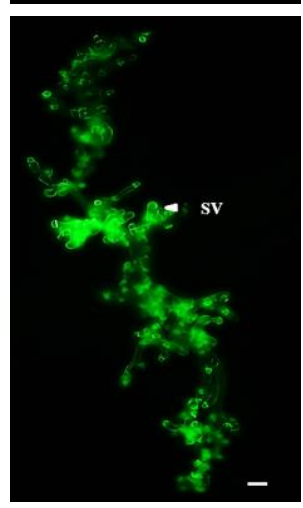

BSMV: TaBln1-1as
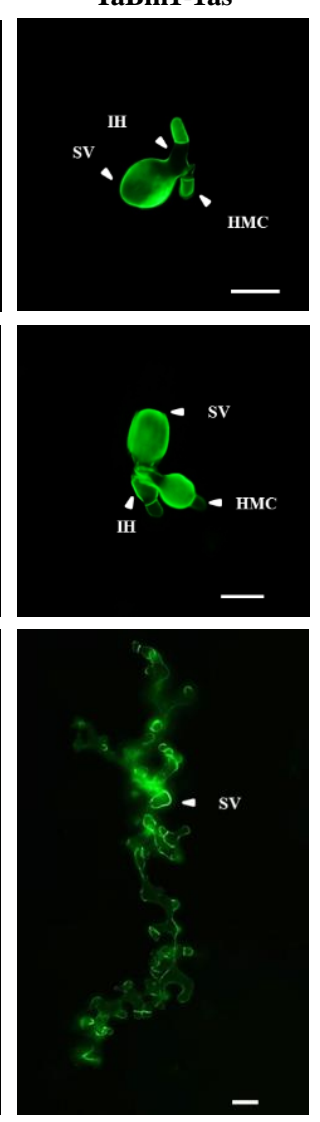

BSMV: TaBIn1-2as
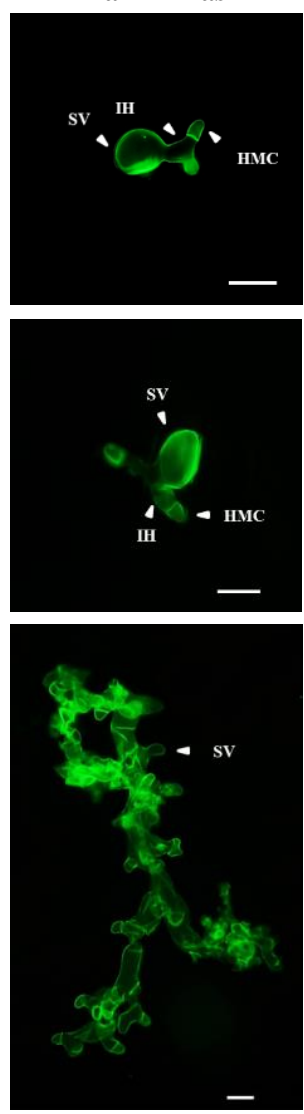

B

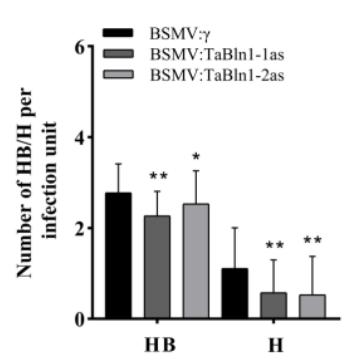

C

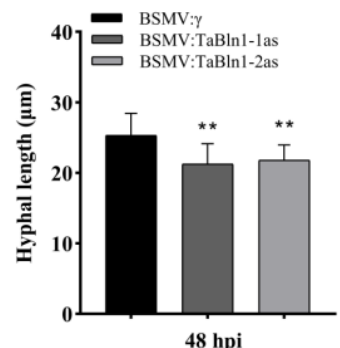

D

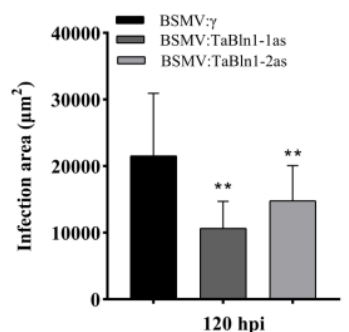

Figure 3. Knocking down TaBln1 reduces growth and development of Pst race

CYR31. A, Fungal structures in wheat leaves infected with BSMV and Pst were visualized with WGA and the fungal structures were observed under a fluorescence microscope. SV, substomatal vesicle; HMC, haustorial mother cell; IH, infection hypha. H, haustoria. B, The average number of hyphal branches (HB) and haustoria (H) of Pst in each infection site were counted at 24 hpi. C, The hyphal length of Pst in TaBlnl-silenced plants at 48 hpi. Hyphal length is the average distance to the tip of the hypha from the intersection of the sub-stomatal vesicle and the hypha was measured using CellSens Entry software (unit in $\mu \mathrm{m}$ ). D, The infection area at $120 \mathrm{hpi}$ was calculated using DP-BSW software (units of $\mu \mathrm{m}^{2}$ ). Values were derived from three biological repetitions (50 infection sites each time). Asterisks indicate a significant difference $(* \mathrm{P}<0.05, * * \mathrm{P}<0.01)$ from BSMV: $\gamma$ inoculated plants using the Student's $t$ test. 
855

856

857

858

859

860

861

862

863

864

865

866

867

868
A

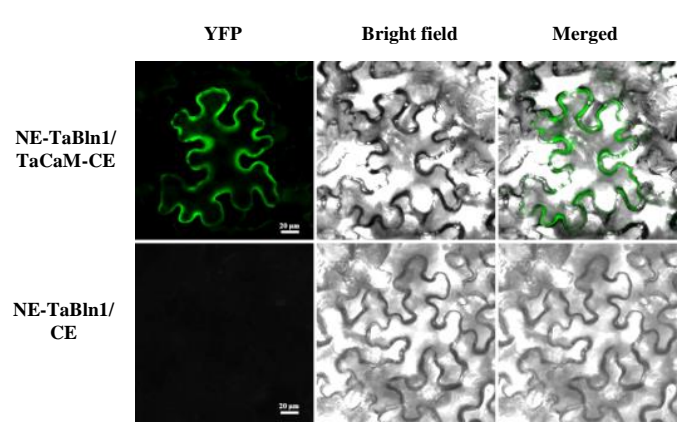

C

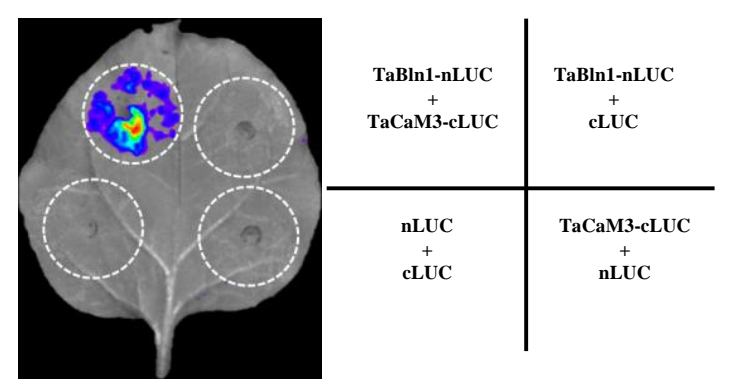

B

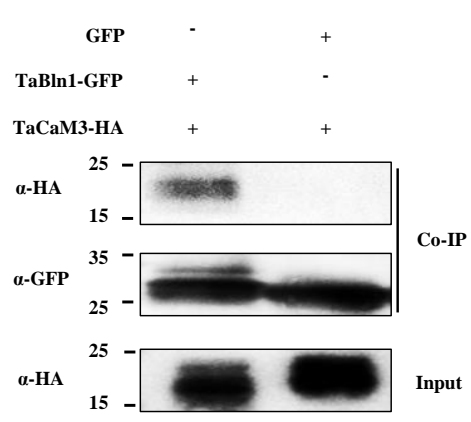

D

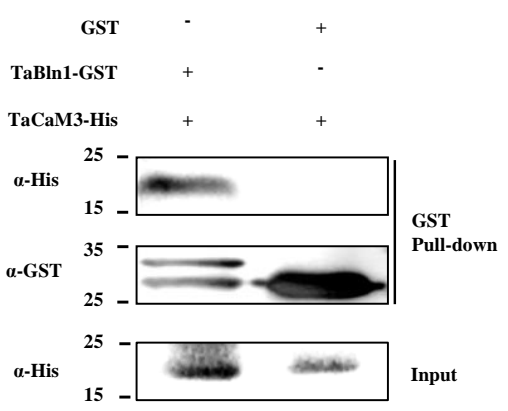

Figure 4. TaBln1 interacts with TaCaM3. A, BIFC confirmed the interaction of TaBlin1 and TaCaM3. NE-TaBlin1/TaCaM-CE is shown in the first panels, and $\mathrm{NE}-\mathrm{TaBlin} 1+\mathrm{CE}$ as negative control is shown in the second panels. Bright-field and YFP fluorescence (in green) images were taken by confocal microscopy. Bar $=20 \mu \mathrm{m}$. B, Protein interaction of TaBln1-GFP and TaCaM3-HA were determined by Co-IP assay. Anti-HA and anti-GFP were used to detect protein expression. GFP was used as a negative control. kDa, kilodaltons. C, Split-luciferase assays demonstrated the interaction of TaBln1 and TaCaM3 in N. benthamiana leaves. The nLUC and cLUC alone were used as negative controls. D, GST pull down assay was used to detect the interaction between TaBln1 and TaCaM3. The recombinant TaBln1-GST and TaCaM3-His expressed in E. coli were subjected to GST pull down analysis. GST was used as a negative control. $\mathrm{kDa}$, kilodaltons. 
bioRxiv preprint doi: https://doi.org/10.1101/2021 07.16.452683; this version posted July 16, 2021. The copyright holder for this preprin (which was not certified by peer review) is the author/funder, who has granted bioRxiv a license to display the preprint in perpetuity. It is made available under aCC-BY-NC-ND 4.0 International license.

A

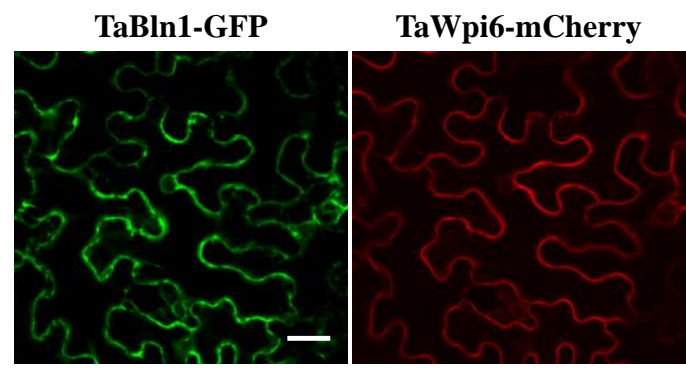

B

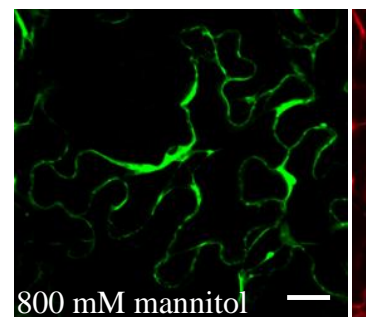

C

TaCaM3-GFP

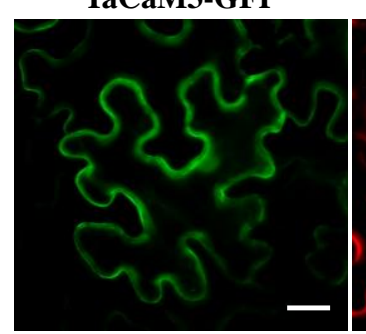

TaBIn1-mCherry

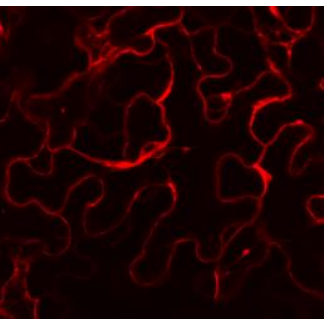

Tabln1-mCherry
Bright field

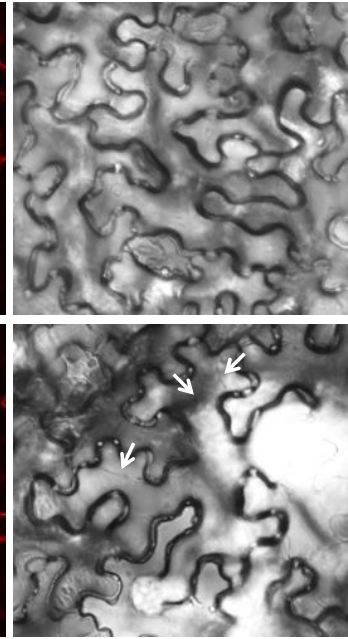

Bright field

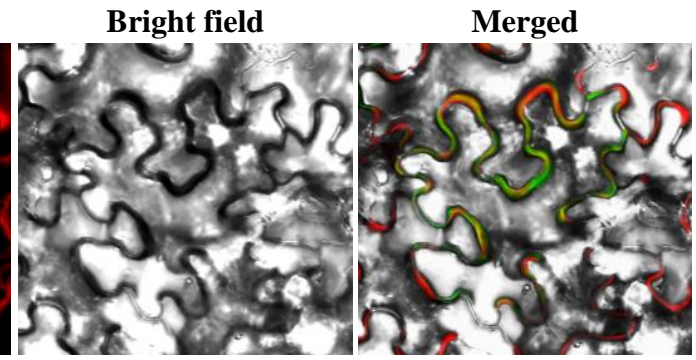

Merged

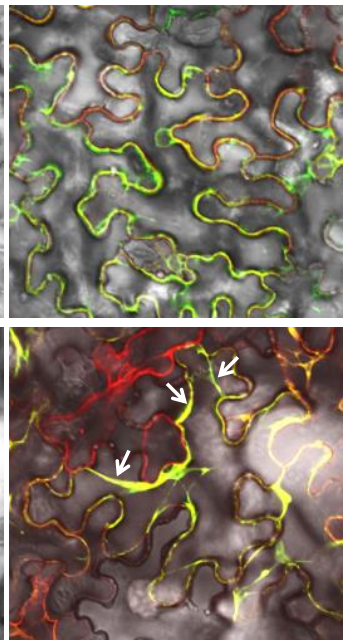

Figure 5. Subcellular localization of TaBln1 and co-localization of TaBln1 and TaCaM3 in $N$. benthamiana leaves. A and B, Co-expression of TaBln1-GFP and TaWpi6-mCherry in $N$. benthamiana leaves. GFP fluorescence is in green; TaWpi6-mCherry (red fluorescence) indicates TaWpi6 labeling the plasma membrane. Leaf epidermal peels in (B) were plasmolyzed (white arrows) by incubation in 800 $\mathrm{mM}$ mannitol for $6 \mathrm{~min}$. C, TaCaM3-GFP and TaBln1-mCherry were co-expressed in $N$. benthamiana leaves. Bright field (BF) images show the equivalent field observed under white light. Merged TaBln1-GFP/TaWpi6-mCherry/Bright field (A and B) and TaCaM3-GFP/TaBln1-mCherry/Bright field (C) images are shown. All of the signals were monitored by confocal microscopy. Bar $=20 \mu \mathrm{m}$. 
A

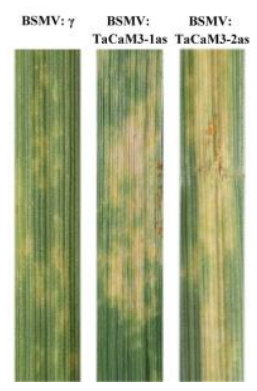

D

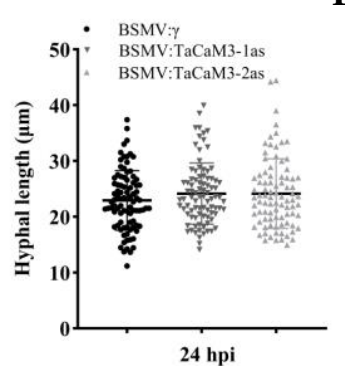

G

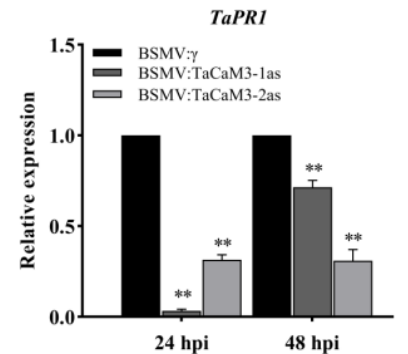

B

$\mathbf{E}$
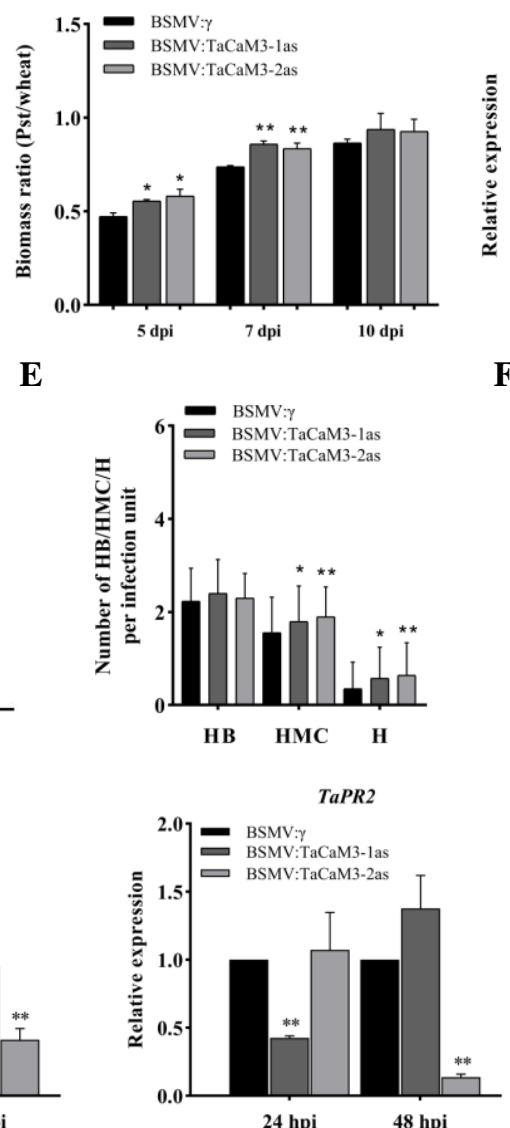

C

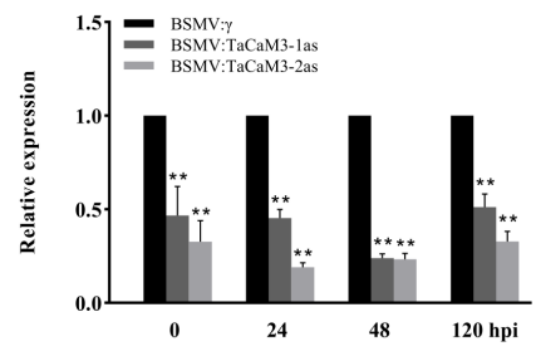

$\mathbf{F}$

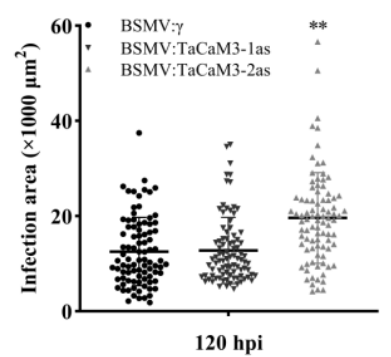

TaPR5

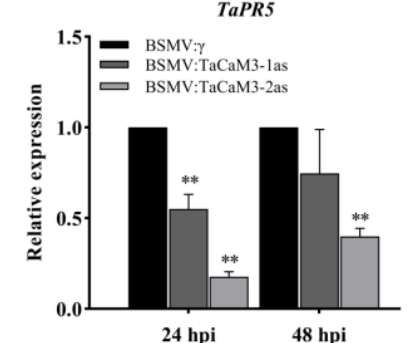

882

883

884

885

886

887

888

889

890

891

892

893

894

895

896

897

898

899

900

901

Figure 6. Silencing $\mathrm{TaCaM3}$ reduces wheat resistance to Pst CYR23. A, Disease phenotypes of the fourth leaves pre-inoculated with BSMV constructs and then challenged with Pst CYR23. B, The biomass ratio (Pst/wheat) measurements of total DNA extracted from BSMV-treated wheat leaves infected by CYR23 at 5, 7 and 10 dpi based on qRT-PCR. Ratio of total fungal DNA to total wheat DNA was assessed by normalizing the data to the wheat gene TaEF-1 $\alpha$ and the Pst gene PstEF1. C, The relative expression levels of TaCaM3 in leaves inoculated with CYR23 were assayed by qRT-PCR at $0,24,48$, and 120 hpi. D, The hyphal length of Pst in TaCaM3-silenced plants at $24 \mathrm{hpi}$. E, Average number of hyphal branches (HB), haustorial mother cells (HMC), and haustoria $(\mathrm{H})$ of Pst in each infection site were counted at 24 hpi. F, TaCaM3-silenced plants show a significant increase in infection unit area at 120 hpi. Means were calculated from 50 infection sites of three biological replicates and were represented as solid lines in the picture. G, Relative expression of the marked defense-related genes in the fourth leaves at 24 and 48 hpi. Transcript levels were quantified by qRT-PCR and normalized with TaEF-1 $\alpha$. The mean and standard deviation were calculated from three independent biological replications. Asterisks indicate a significant difference $(* \mathrm{P}<0.05, * * \mathrm{P}<0.01)$ from BSMV: $\gamma$ inoculated plants using the Student's $t$ test. 
A

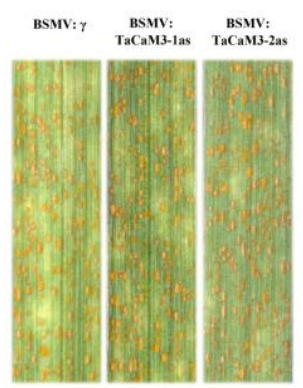

C

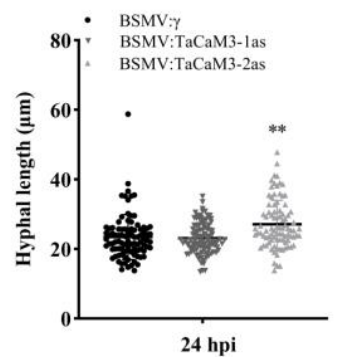

B

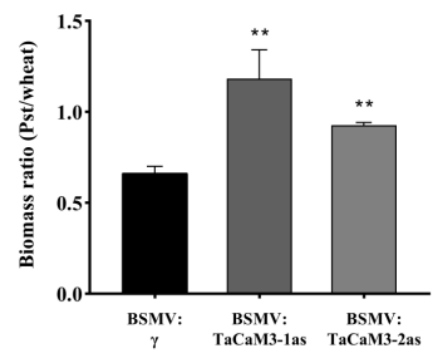

D

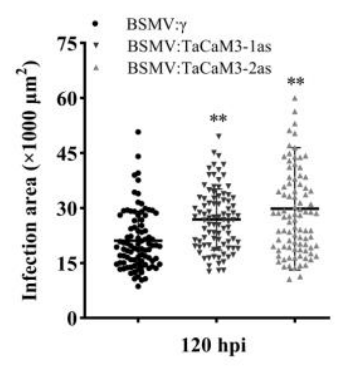

903

904

905

906

907

908

909

910

911

912

913

Figure 7. Knocking down TaCaM3 enhances wheat susceptibility to Pst CYR31. A, Disease phenotypes of the fourth leaves pre-inoculated with BSMV constructs and then challenged with Pst CYR31. B, The biomass ratio (Pst/wheat) measurements of total DNA extracted from BSMV-treated wheat leaves infected by CYR31 at 5 dpi based on qRT-PCR. C, Significant increase in the hyphal length of Pst in TaCaM3-silenced plants at 24 hpi. E, TaCaM3-silenced plants show a significant increase in infection unit area at 120 hpi. Means were calculated from 50 infection sites of three biological replicates and were represented as solid lines in the picture. Asterisks indicate a significant difference $(* * \mathrm{P}<0.01)$ from BSMV: $\gamma$ inoculated plants using the Student's $t$ test. 
914

915

916

917

918

919

920

921

922

923

924
A

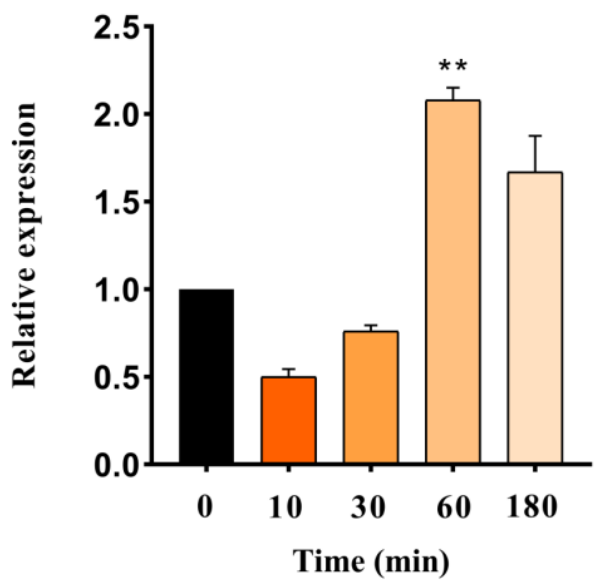

B

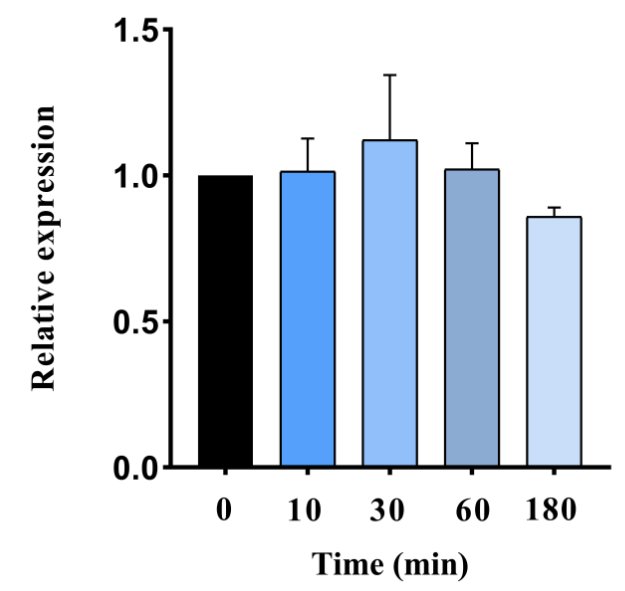

Figure 8. TaCaM3 expression changes in response to two PAMPs (chitin and flg22) treatment. A, The expression levels of TaCaM3 in leaves treatment with chitin were assayed by qRT-PCR at 0, 10, 30, 60 and $180 \mathrm{~min}$ post treatment. B, The expression levels of TaCaM3 in leaves treatment with flg22 were assayed by qRT-PCR at 0, 10, 30, 60 and 180 min post treatment. Expression levels were normalized to TaEF-1 $\alpha$. The mean and standard deviation were calculated from three independent biological replications. Asterisks indicate a significant difference $(* * \mathrm{P}<$ 0.01 ) from control (water treatment) using the Student's $t$ test. 
A

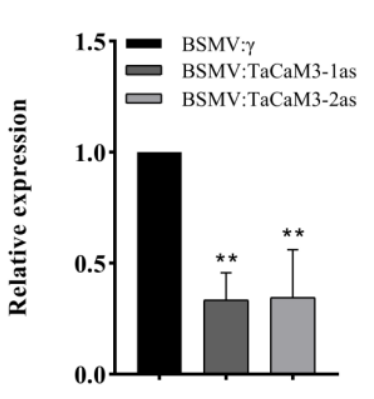

C

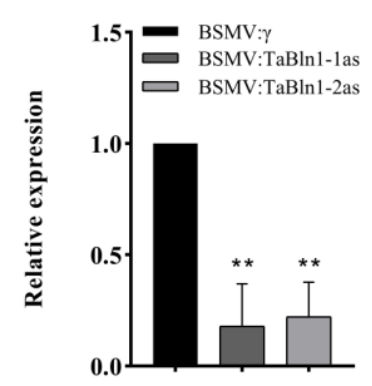

B

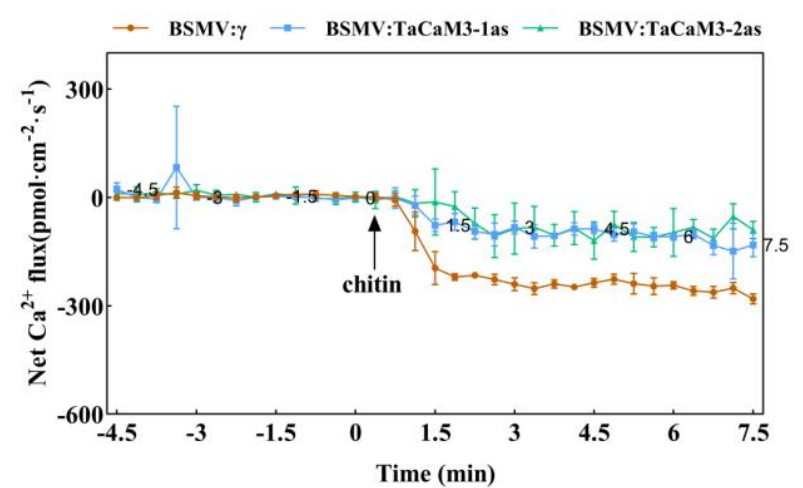

D

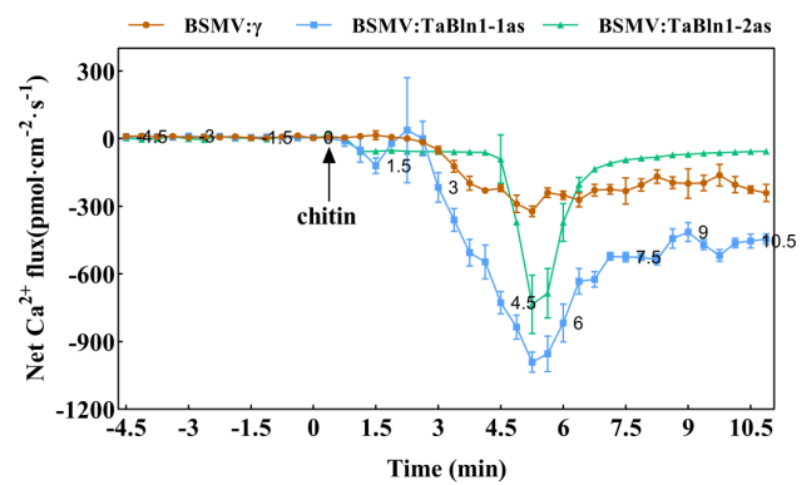

Figure 9. $\mathrm{Ca}^{2+}$ signaling in response to chitin in $\mathrm{TaBln1}$ - and $\mathrm{TaCaM3}$-knockdown plants. A, The expression of TaCaM3 was substantially knocked down by VIGS. B, Comparison of calcium influx in mesophyll cells from BSMV: $\gamma$ inoculated plants and BSMV:TaCaM3 inoculated plants after chitin treatment. C, The expression of TaBln1 was substantially knocked down by VIGS. D, Comparison of calcium influx in mesophyll cells from BSMV: $\gamma$ inoculated plants and BSMV:TaBln1 inoculated plants after chitin treatment. Transcript levels were quantified by qRT-PCR and normalized with TaEF-1 $\alpha$. The relative expression of TaBln1 and TaCaM3 were calculated using the comparative threshold method $\left(2^{-\Delta \Delta C T}\right)$. Values were derived from three biological repetitions. Asterisks indicate a significant difference $(* * \mathrm{P}<0.01)$ from BSMV: $\gamma$ inoculated plants using the Student's $t$ test. 
A

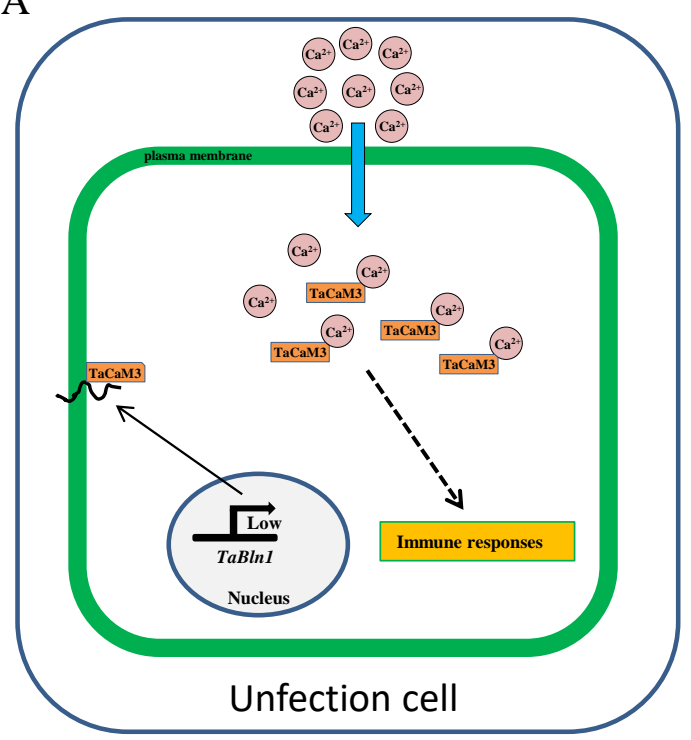

$\mathrm{B}$

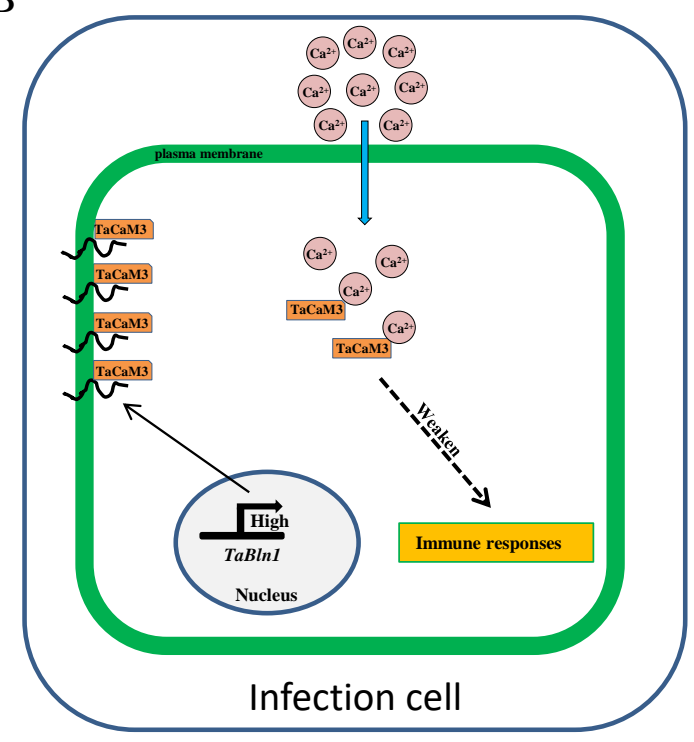

Figure 10. The proposed model of the function of $T a B \ln 1$ in the wheat-Pst interaction. A, In uninoculated wheat leaves (Figure 10A), the expression of $\operatorname{TaBln} 1$ is maintained at a low level. TaCaM3 functions normally in binding $\mathrm{Ca}^{2+}$, and it may be transferred to downstream targets to activate the immune responses. During Pst infection of wheat (Figure 10B), the expression of TaBln1 may be upregulated and localized to the plasma membrane. The interaction between $\mathrm{TaB} \ln 1$ and $\mathrm{TaCaM} 3$ results in the accumulation of $\mathrm{TaCaM} 3$ on the plasma membrane. This, in turn, affects the balance between calcium binding and calcium influx, thereby reducing the transmission of $\mathrm{Ca}^{2+}$ signals to downstream targets, and ultimately weakening the disease resistance of wheat. 